\title{
A Profiler for a Heterogeneous Multi-Core Multi-FPGA System
}

\author{
by
}

Daniel Pereira Nunes

A thesis submitted in conformity with the requirements

for the degree of Master of Applied Sciences

Graduate Department of Electrical and Computer Engineering

University of Toronto

Copyright (C) 2008 by Daniel Pereira Nunes 


\title{
A Profiler for a Heterogeneous Multi-Core Multi-FPGA System
}

\author{
Daniel Pereira Nunes \\ Master of Applied Sciences \\ Graduate Department of Electrical and Computer Engineering \\ University of Toronto
}

2008

\begin{abstract}
The TMD is a heterogeneous multi-FPGA system that uses the Message Passing Interface (MPI) programming model to build systems comprised of processors and hardware engines implemented on FPGAs, all interacting using MPI messages. This thesis implements a profiling system that can monitor communication calls and user states. The data is compatible with Jumpshot, a visualizer already used for software-based MPI applications. We demonstrate the capability of the profiler on a number of applications and show that the profiler can be used for multiple FPGAs across multiple boards.
\end{abstract}




\section{Acknowledgements}

First and foremost I would like to thank my supervisor Professor Paul Chow for his guidance and technical advice which were of highest value throughout the course of this MASc. I would also like to thank Emanuel and Alex, for their suggestions and conversations; Manuel, Arun and Chris for their expertise advice; and all the remaining group members: Andrew, Danny, David, Ali, Keith, Daniel Ly, Danyao, Richard, Tom, Mike, Nasim, and Matt for their support. Last but not the least, Professor João Cardoso who first contacted the University of Toronto and provided guidance throughout all my undergrad in Portugal. 


\section{Contents}

1 Introduction 1

1.1 Motivation . . . . . . . . . . . . . . . . . . . . . 1

1.2 Research Contributions . . . . . . . . . . . . . . . . . 2

1.3 Thesis Organization . . . . . . . . . . . . . . . . . 3

2 Background 4

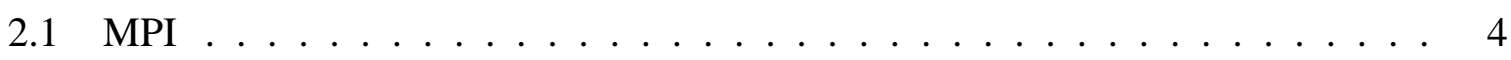

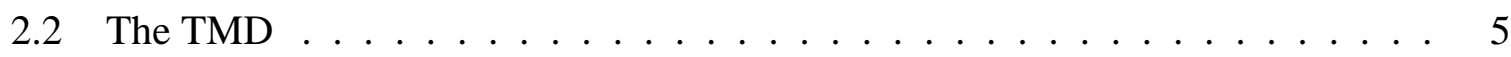

2.2.1 Computation nodes . . . . . . . . . . . . . 7

2.2.2 Off-Chip Communications Nodes . . . . . . . . . . . . . . . 7

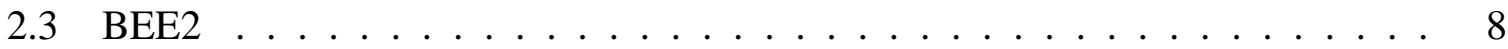

2.4 Performance Analysis for Reconfigurable Computers . . . . . . . . . . . . 8

2.5 Related Work . . . . . . . . . . . . . . . . . . . . . . . . 10

3 Profiler 12

3.1 Hardware . . . . . . . . . . . . . . . . . . . . 12

3.2 Clock Counter Synchronization . . . . . . . . . . . . . . . 15

3.3 Software . . . . . . . . . . . . . . . . . . . . 17

4 Case Studies $\quad 19$

4.1 Barrier . . . . . . . . . . . . . . . . . . . . . 19

4.2 The Heat Equation Application . . . . . . . . . . . . . . . . . . . 22

4.3 The LINPACK Benchmark . . . . . . . . . . . . . . . . . . . . . . . . 26

4.4 TMD-MPE - Unexpected Messages Queue . . . . . . . . . . . . . . . . . . 32

5 Profiler Overhead $\quad 35$

6 Profiler Implementation on Other Platforms 37

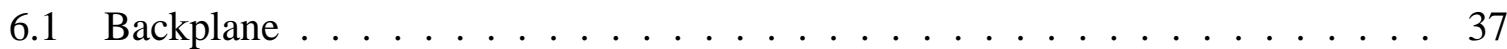


6.2 HPRC Implementation Platform . . . . . . . . . . . . . . . . . . . . 39

7 Conclusions $\quad 41$

8 Future Work 43

9 Appendixes $\quad 45$

9.1 Appendix A . . . . . . . . . . . . . . . . 45

9.1.1 Example System . . . . . . . . . . . . . . . . . 45

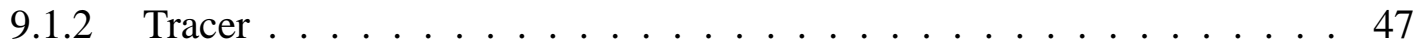

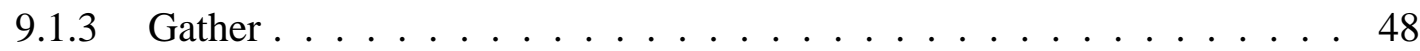

9.1 .4 Collector .......................... 49

$\begin{array}{ll}\text { Bibliography } & 51\end{array}$ 


\section{List of Tables}

4.1 State's Duration . . . . . . . . . . . . . . . . . . . . . 29

5.1 Tracers Overhead . . . . . . . . . . . . . . . . 36

9.1 MPE_Tracer's Interface . . . . . . . . . . . . . . . . . . . 47

9.2 Gather Interface . . . . . . . . . . . . . . . . . . . . . . . 48

9.3 Collector Interface $\ldots \ldots \ldots$. . . . . . . . . . . . . . . . . . . 49 


\section{List of Figures}

2.1 TMD Architecture $\ldots \ldots \ldots \ldots \ldots$

2.2 BEE2 Architecture . . . . . . . . . . . . . . . . . 8

3.1 High-Level Profiler Architecture . . . . . . . . . . . . . . . . . 13

3.2 a) Processor Profiler Architecture b) Hardware Engine Profiler Architecture . . 14

3.3 a) Communication Tracer b) Computation Tracer (Processor) c) Computation Tracer (Engine) . . . . . . . . . . . . . . . . . . . . . 16

4.1 Barrier Communication Patterns . . . . . . . . . . . . . . 20

4.2 Barrier implemented sequentially . . . . . . . . . . . . . . . 21

4.3 Barrier implemented as a binary tree . . . . . . . . . . . . . . . 21

4.4 Data decomposition and point-to-point communication in Jacobi algorithm . . 23

4.5 Using blocking calls . . . . . . . . . . . . . . . . . 25

4.6 Using non-blocking calls . . . . . . . . . . . . . . . . 25

4.7 Linpack Benchmark Testbed . . . . . . . . . . . . . . . . . . . . 28

4.8 Linpack Benchmark Iteration . . . . . . . . . . . . . . . . . . . . 30

4.9 Linpack Benchmark General View . . . . . . . . . . . . . . . . . . . 31

4.10 Barrier implemented sequential with TMD-MPE profiled . . . . . . . . . . . 33

6.1 The Backplane . . . . . . . . . . . . . . . . . . . . 38

6.2 Possible Implementation of the Profiler on the Backplane . . . . . . . . . . . . 39

6.3 Possible Implementation of the Profiler on the HPRC Platform . . . . . . . . . 40

9.1 Example system . . . . . . . . . . . . . . . . . . . . 46 


\section{Glossary}

This glossary contains some of the acronyms used in this thesis.

BEE2 Berkeley Emulation Engine 2

CE Computing Element

DMA Direct Memory Access

FIFO First-In-First-Out

FSL Fast Simplex Link

GPIO General Purpose Input/Output

MGT. MultiGigabit Transceiver

MPI Message Passing Interface

NetIf Network Interface

TMD. Originally meant Toronto Molecular Dynamics machine, but this definition was rescinded as the platform is not limited to Molecular Dynamics. The name was kept in homage to earlier TM-series projects at the University of Toronto.

XAUI X-Attachment Unit Interface 


\section{Chapter 1}

\section{Introduction}

This chapter provides details of the motivation of this thesis in Section 1.1. Section 1.2 outlines the main contributions of this work, and Section 1.3 presents a summary of the content of the remaining chapters of this thesis.

\subsection{Motivation}

Understanding the behavior of computer applications without the aid of program analysis tools can become a tedious, and sometimes impossible, task. Computer applications range from simple sequential codes, where an analysis of the current position within the source code may be sufficient to understand it, to complicated parallel codes running on heterogeneous systems that require not only the position of each processor within its source code but also a time relationship between them. A profiler goal is to give understanding of the application while requiring the smallest effort from the programmer and without affecting the system significantly.

Field-Programmable Gate Array (FPGA) reconfigurability allows us to gain performance by creating application-specific hardware. Some high-performance computing platforms now include FPGAs that can be plugged directly into an Intel Front Side Bus (FSB) [1] socket or an AMD Opteron [2] socket using the hyper-transport protocol. Some systems rely only on FPGAs, taking advantage of their embedded cores (e.g. PowerPC [3]) for general computation 
and specialized hardware engines for the more demanding computation. However, failing to have a complete understanding of the application may result in underachieved performance. To address this, FPGA reconfigurability may also be used to create a custom profiler that will provide the knowledge needed to configure the hardware system for a specific application.

This thesis focus is a profiler for the TMD [4] [5] [6], a heterogeneous multi-core multiFPGA system designed at the University of Toronto that uses the message passing paradigm for communications. The main purpose of this profiler is to give insight into the communications occurring between nodes and the computation performed by each node. The presence of different cores in the system increases the difficulty of profiling, since the profiler is required to be compatible with all of them. Furthermore, the possibility of having hardware engines introduces the need of having the profiler's functionality implemented both in software and hardware.

\subsection{Research Contributions}

The contributions of this thesis are derived from the integration of ideas already used in other systems (e.g. traditional clusters) into a new multi-core multi-FPGA system, the TMD. Due to the different nature of traditional clusters and the TMD, the implementation of such ideas differ completely "under the hood", but provide the opportunity for users to profile their applications using interfaces already familiar to them. Following the same easy-to-port philosophy of the TMD, users are able to port their already instrumented source code from a cluster into the TMD in a seamless way.

The profiler infrastructure provides a first attempt of profiling these systems and can be used as a working platform for future students at the University of Toronto that wish to research further into performance analysis. 


\subsection{Thesis Organization}

The remainder of this thesis is organized as follows: Chapter 2 provides background information to better understand the contents of this thesis. Chapter 3 describes the profiler implementation. In Chapter 4 the profiler is tested with four case studies. Then in Chapter 5 the profiler overhead is considered. Chapter 6 considers the implementation of the profiler on other implementations of the TMD. Finally, Chapter 7 concludes and in Chapter 8 ideas are presented for future work.

In the appendix there is helpful information on how to include the profiler on a hardware system. 


\section{Chapter 2}

\section{Background}

This chapter presents a brief overview of key concepts to better understand this work, and to establish a context for the ideas presented in this thesis. Each section describes parts of related knowledge and presents some definitions that will be used in the rest of this document. Section 2.1 is a brief introduction to the known message passing library, MPI [7] [8]. Section 2.2 and Section 2.3 describe the hardware system on which the profiler was applied. Section 2.4 introduces the challenges of performance analysis for reconfigurable computers. Section 2.5 examines related work in the field of profiling, both in sequential and parallel applications, and traditional clusters and FPGA systems.

\subsection{MPI}

Message passing has become a common programming model used for distributed memory computing systems. Message passing assumes that each process contains the necessary data in local memory and that no direct access to global memory is available. Transactions between processes are defined explicitly by the programmer, which is also responsible for the execution's correctness. MPI, the best known message passing library, provides a language and architecture independent communication protocol that is highly scalable and portable. It was created in 1992 and is currently maintained by the MPI Forum [7]. The API is organized 
in layers built on top of each other. While the lower layers are hardware dependent and need to be adapted for different architectures, the upper layers are standard and allow the user code to be usable across different platforms. This portability is one of the main reasons for the success of MPI. Communications can be done by point-to-point or collectively calls. Point-to-point calls, MPI_Send and MPI_Recv, are use to send and receive data, respectively. Collective calls are built on top of point-to-point calls simplifying the user code by defining important group communication. Examples of collective calls are MPI_Barrier, a synchronization routine, and MPI_Broadcast, a routine where one process broadcasts data to all the others.

The communication protocol can be either synchronous or asynchronous. The Rendezvous protocol is a synchronous communication mode where a process will only initiate a transfer when the receiver acknowledges the operation. The transfer is initiated by the producer process sending a request to send packet composed of its rank, a destination rank, size of message and a 32-bit tag, which is a user-defined message identificator. The Eager protocol is an asynchronous protocol communication mode where a process will initiate a transfer without consulting the other process. In this communication mode, the producing process assumes that enough buffering exists to hold its message at the receiver.

Each process is represented by a unique integer, called the rank, spanning from 0 to size-1, where size is the total number of processes on the system.

\subsection{The TMD}

The TMD is a heterogeneous multi-core, multi-FPGA system developed at the University of TMD that achieves high performance by taking advantage of hardware acceleration using FPGAs. The system can be fully customized to a specific application by adapting the on-chip network topology and choosing appropriate computation elements for each task. The possibility of having heterogeneous cores requires a flexible communication protocol that is not dependent on any particular computation element, and that simultaneously allows the seamless 
addition, removal or exchange of cores to better fit a particular application. This flexibility co-exists in this system both in the hardware level, where a node can be physically removed without changing the rest of the system, and in the software level where the source code of an application is independent of the number and nature of nodes.

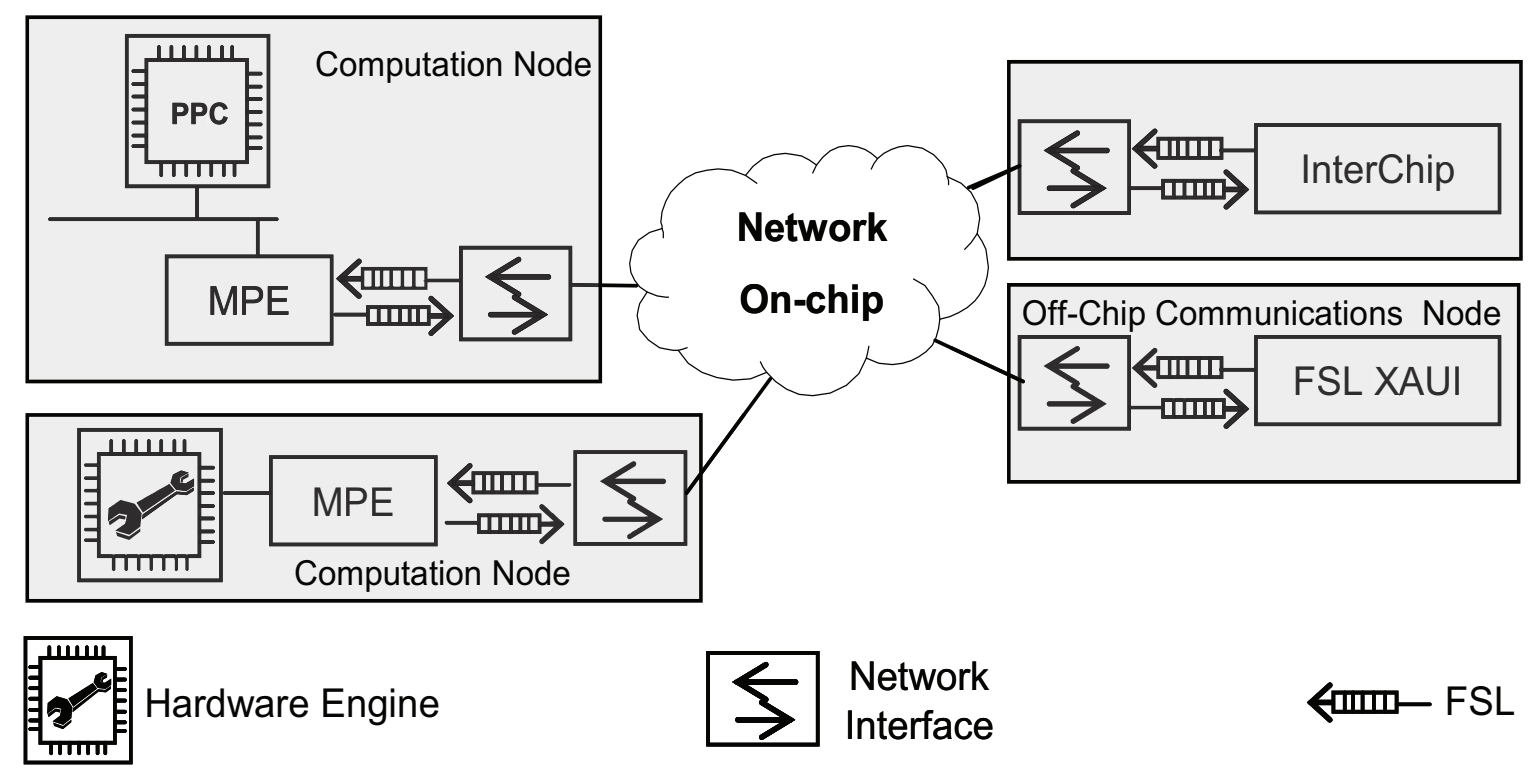

Figure 2.1: TMD Architecture

TMD-MPI [5] is a lightweight implementation of MPI for embedded processors in a heterogeneous multiprocessor system using the Rendezvous protocol. The nodes can be divided into two types: computation nodes, that are responsible for all computation, and network nodes that route message packets outside of the FPGA. All nodes interact with the on-chip network through a Network Interface (NetIf) core that contains the routing tables. Communications between all hardware blocks are done through Xilinx's Fast Simplex Links (FSLs), which are unidirectional FIFOs that provide isolation between the Computation Elements (CEs), allowing different clock domains, while providing buffering for ongoing messages. Fig. 2.1 shows all types of nodes that can exist in the TMD. 


\subsubsection{Computation nodes}

Computation can be performed by a Microblaze, a PowerPC or a hardware engine. Because the application source code can be kept constant from platform to platform, due to the TMD-MPI abstraction, a Microblaze or a PowerPC can be used as an intermediate stage when porting an application from a traditional cluster to the TMD. After the application is successfully ported in software, if the task running on one of these processors is critical it may then be optimized by substituting it with a hardware engine. Because all communications are done using the TMD-MPI protocol, a special core that encapsulates the TMD-MPI functionality, the TMDMessage Passing Engine (TMD-MPE) [6], was designed so all hardware engines can send and receive messages from other nodes in full-duplex mode. This simplifies significantly the design process of a hardware engine, as the designer will not need to be concerned with the details of the protocol such as unexpected messages and packetization. Although the TMD-MPE was mainly designed to be used by engines, it can also be used by a Microblaze or a PowerPC, reducing the memory footprint of their code and, for the processors, increasing communication speed by allowing Direct Memory Access (DMA).

\subsubsection{Off-Chip Communications Nodes}

Off-chip communications nodes do not perform any computation and are present on the system to route message packets outside to another FPGA. Their configuration depends on which platform the TMD is being implemented on. This paper will only consider the Berkeley Emulation Engine 2 (BEE2) [9] boards for implementation (see Section 2.3 ). There are two possible types of off-chip communications on the BEE2 board implementation: intra-board and inter-board. The intra-board communications refer to messages sent between nodes from different FPGAs on the same board and are done using direct connection wires between the FPGAs, through the Interchip core. The inter-board communications refer to communications performed by different nodes in different boards and are done using the X Attachment Unit Interface (XAUI) standard. As with all blocks, the interface between the on-chip network and XAUI is done 
using the FSL standard.

\subsection{BEE2}

The BEE2 platform contains five Xilinx Virtex II Pro 70 FPGAs per board with 4 GB per FPGA of high speed DDR DRAM. The FPGAs are connected in a star topology, with the Control FPGA being the central node, and a ring topology between the User FPGAs. Each User FPGA has four 10Gbps links, configured as XAUI links in our applications, while the Control FPGA has two. The Control FPGA is also connected to common I/Os such as the 10/100 ethernet, USB 1.1, RS232 serial and DVI.
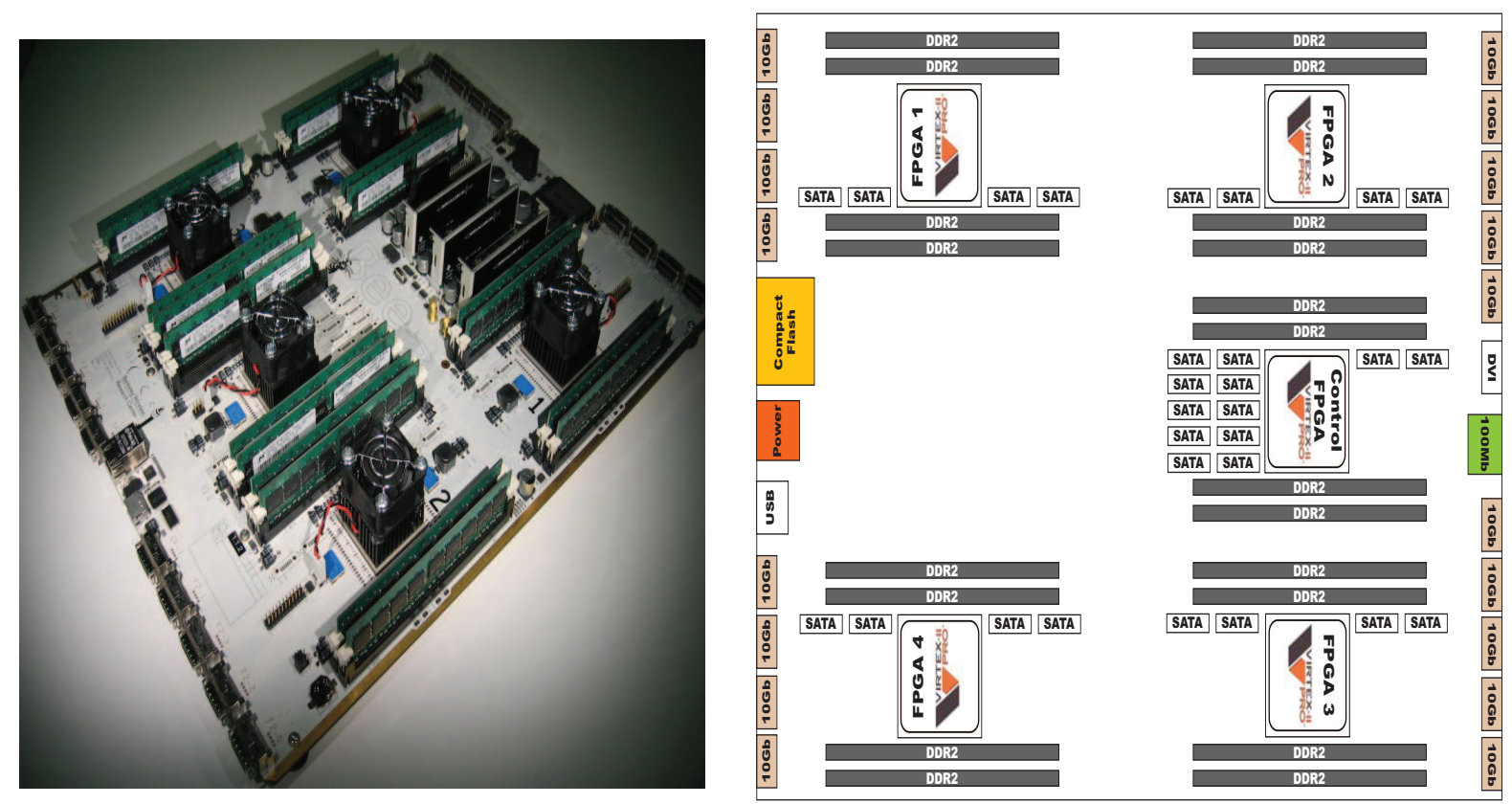

Figure 2.2: BEE2 Architecture

\subsection{Performance Analysis for Reconfigurable Computers}

Performance analysis of reconfigurable computing comes with significant challenges [10]. Such challenges include perturbing as little as possible the original application's behaviour, while retrieving sufficient data so that the behavior can be reconstructed; and providing the 
flexibility to analyze different applications, while requiring little effort from the designer. What data to instrument plays an important role in reconfigurable computers analysis. Possible causes of performance bottlenecks in hardware are not as well determined as they are in software, and the immense number of hardware systems makes it harder to have a common bottleneck guide. Nevertheless, communications, off-chip (e.g. FPGA, CPU, main memory), on-board (e.g. FPGA, DDR) or on-chip (e.g. CE), are a known source of bottlenecks. However, instrumenting all communications can become a challenge due to the amount of parallelism inside a FPGA. The TMD-MPI (see Section 2.2) in conjunction with the TMD-MPE and the FIFO-based on-chip network, provides an excellent abstraction for the communications. This abstraction allows not only running an application as it would be run in a traditional cluster, but also to profile it as such. This is, the FPGA is not profiled as one uniform core, but as multiple independent cores. Another important step prior to instrumentation is to decide at what level that instrumentation should be performed. Instrumentation can be performed either at a source-level or binary-level. Although other intermediate levels may exist, the fact that some only exist in memory during synthesis and implementation, and their documentation is poor, reduces the advantages of using them and are out of the scope of this work. Binary-level instrumentation provides a fast method of performing changes on the application since it occurs after place-and-route. Also, it's portable across languages for a specific device. However, instrumenting a bitstream is extremely hard. Not all data is accessible, and correlation with the source will be impossible most of the time since synthesis and implementation significantly transform the system. Source-level instrumentation allows correlation with the source, it's easier to implement and portable across devices. The largest setback is the number of hours it may take to instrument since any change to the system at source-level requires the system to be built again. 


\subsection{Related Work}

A large amount of research has been done to create or improve profilers. Profiling data can be gathered from hardware performance counters, that collect low level performance metrics (e.g. clock cycles, instruction counts, cache misses) or from the source code itself by instrumenting it. In 1979, Unix systems included a tool called prof that would list each function and give the number of calls and time spent on them. Gprof [11] was released in 1982 and included a call graph analysis that would provide not only the time spent in each function but also how much time each function spent on behalf of each of its callers. The Performance Application Programming Interface (PAPI) [12] gives access to hardware performance counters on modern microprocessors allowing the monitoring of high-level hardware events.

For parallel systems, such as multicore processors, profilers that can relate events over different processors and provide information on the interactions of different processing units during a parallel application execution are needed.

Reconfigurable systems can be more challenging to profile than typical clusters of computers. The profilers available tend to gather data from the perspective of a CPU. However, FPGA systems may not have any conventional processor and will most certainly contain hardware engines that will require a more hardware-aware profiler. Techniques like Xilinx ChipScope [13] and Altera's SignalTap [14] have been developed to extract data out of an FPGA with little impact on performance. However, these tools were designed for hardware debugging and are far from adequate to be used as profilers.

The Snooping Software Profiler (SnoopP) [15] is a non-intrusive, real time, profiling tool developed at the University of Toronto. The profiler is able to count how many cycles an application running on a soft-core processor spent in a specific program counter (PC) address region.

The MPI Parallel Environment [16] provides the ability to profile different sections of MPI code, including communications performed between nodes, through instrumentation of the source code. However, it is an intrusive profiler that will add close to $5 \%$ overhead to a typ- 
ical application [17]. It includes a tool called JumpShot [16] that visualizes the profiler logs, allowing a user to see what each process is doing over a common timeline. The logs created by the profiler presented in this paper are compatible with the logs created by the MPI Parallel Environment. Moreover, it uses the same Application Programming Interface (API) to instrument the source code. This was done because the MPI Parallel Environment is part of the MPI distribution MPICH [16] and will allow us to easily port profiled applications from a traditional cluster to the TMD. Also, Jumpshot is an easy to use visualization tool that is able to open considerably large profiler logs. 


\section{Chapter 3}

\section{Profiler}

The reprogrammable nature of the FPGAs can be used in our favour to create a custom profiler for an application that will minimally impact the system performance, therefore, retrieving the real behavior of the system.

This profiler retrieves data from all TMD-MPI communication calls and computation states defined by the user, both from a processor or a hardware engine, and allows the visualization of all ranks on a timeline. Hardware is profiled by sampling specific hardware events. Software is profiled by embedding code into the MPI functions or using additional profiling functions.

\subsection{Hardware}

The profiler hardware should occupy minimal FPGA real estate and not affect timing by making the application run at a slower clock rate. Unfortunately, these are conflicting goals and a compromise had to be made. We incurred extra hardware to prevent performance loss whenever there was a choice.

One of the most critical points of a profiler is where to store data retrieved from the system and how to redirect it to that location. Because the BEE2 board has considerable memory (4GB of DDR per FPGA), each BEE2 board stores the profiling data of their own FPGAs on the Control FPGA until the end of the computation, when the data is sent to a remote workstation. 


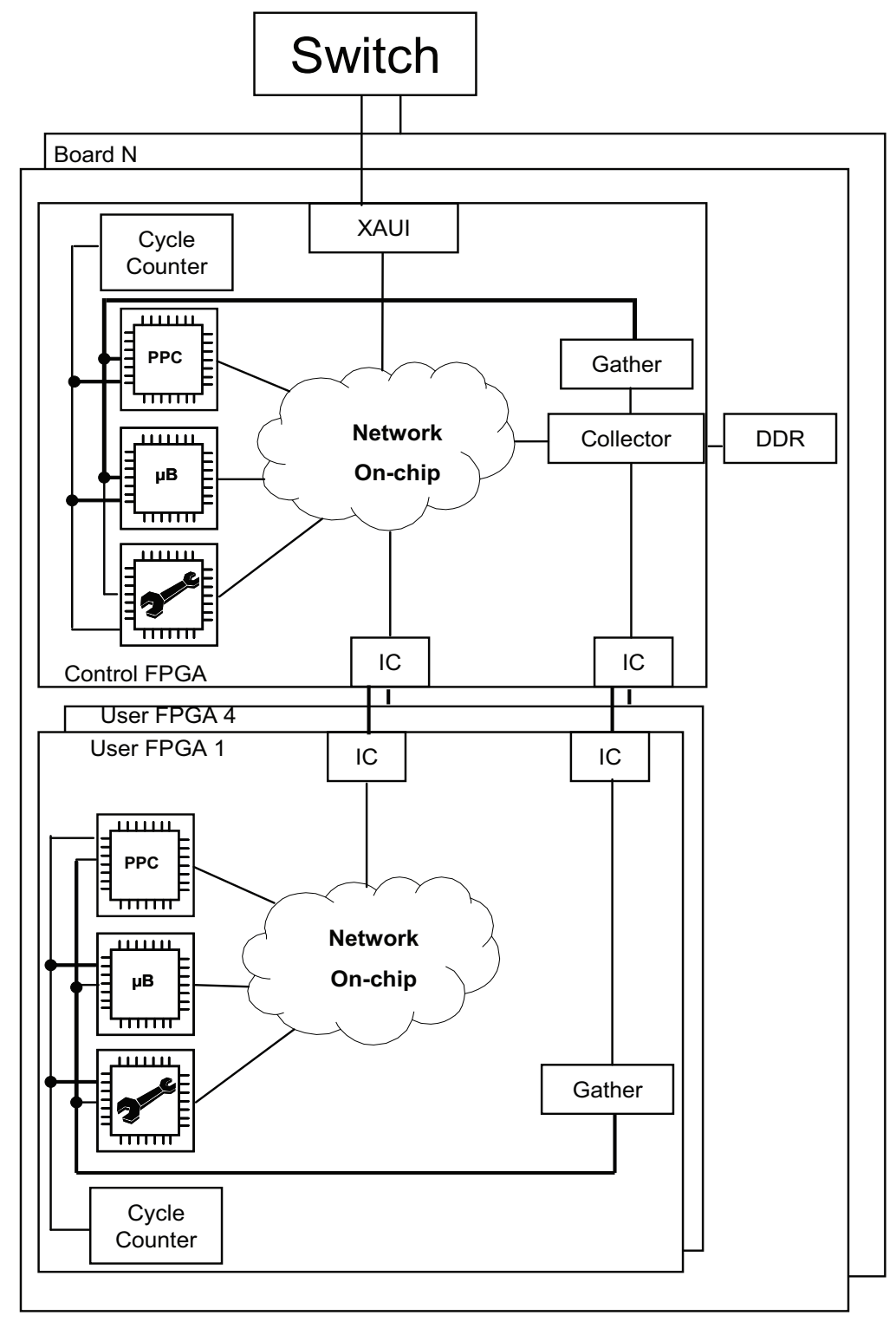

Figure 3.1: High-Level Profiler Architecture

Furthermore, the profiling data does not use any of the communication channels used by the application, but a special network created exclusively for the profiler.

Fig. 3.1 shows the high-level profiler architecture between a User FPGA and a Control FPGA, and Fig. 3.2 shows the connections between the profiler and the computation nodes. Each FPGA contains a 64-bit clock cycle counter, shown in Fig. 3.1, synchronized between all FPGAs. This counter serves as a global timer and is used to obtain the timestamps of events of interest on the system. The Tracers in Fig. 3.3 are the connection point between 
the application and the profiler, and each node will have up to three Tracers, depending on whether the computation is being profiled or not. This number of Tracers is needed because of the parallel nature of hardware where at any given moment a node could be simultaneously computing, sending and receiving. This may also be true for a PowerPC or Microblaze when using a TMD-MPE unit.

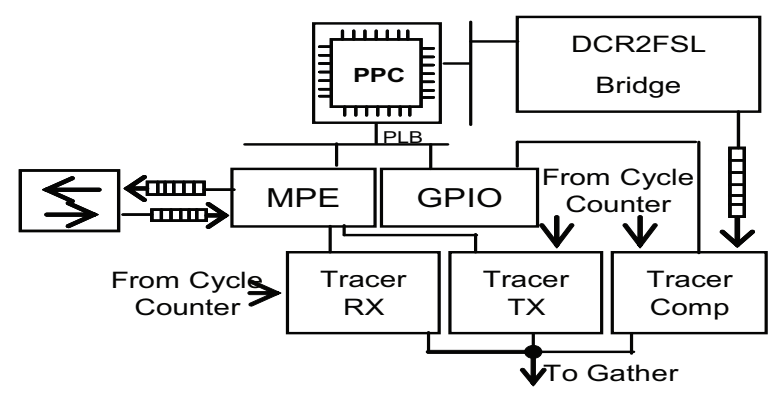

a)

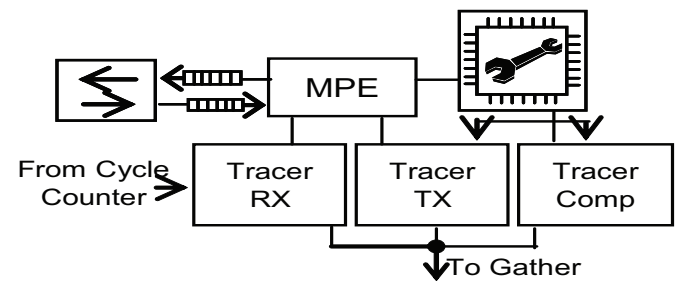

b)

Figure 3.2: a) Processor Profiler Architecture b) Hardware Engine Profiler Architecture

The profiling data of a given instance of a state is kept in the Tracer until that instance finishes. Only then is the data sent, as one packet through the profiler network. This is done to prevent inconsistencies in the profiler data by assuring that either all data referenced to an instance is saved in memory, or that instance is completely discarded.

The Tracers are non-intrusive with the exception of when they are used on a processor, since communication from the processor to the tracer is done through an FSL and General Purpose Input/Output (GPIO), delaying the main computation on the processor. However, when profiling only the computation, the overhead is not significant due to the computation time itself.

The communication Tracers are designed specially for the TMD-MPE. They record all headers, tags and timestamps of the start and end of both the data transfer, request to send 
packets and acknowledge packets in their registers and FIFO, shown in Fig. 3.3 a). The computation Tracers, shown in Fig. 3.3 b), only register the timestamps of the start and end of user-defined states, except when used in conjunction with a processor (e.g.MicroBlaze, PowerPC) without TMD-MPE, where they will also register all MPI calls. For the latter case, the computation Tracers have one set of registers for the MPI calls, and one set of registers and two stacks for the user-defined states. The two sets of registers allow profiling of MPI calls using user-defined states and the two stacks allow nested user-defined states. Sampling of events of interest in the hardware engines is done by computation Tracers built specially for each specific hardware engine. Fig. 3.3 c) shows a generic version of such a tracer capable of fulfilling the profiling needs of most engines.

All Tracers connect to the Gather unit, shown in Fig. 3.1, that will serve as a multiplexor to send the data to the Control FPGA. On the Control FPGA side, the Collector core, shown in Fig. 3.1, will receive data from the other FPGAs and from any core being profiled in the Control FPGA, and will write it to the DDR.

Instrumentation of the hardware is all done at the HDL level. As of now, Tracers and all cores related with the profiler are manually introduced by the user.

\subsection{Clock Counter Synchronization}

Synchronization between FPGAs on the same board is done by releasing their reset signals simultaneously. This can be achieved by having two separate reset signals on the Control FPGA: one to reset all logic needed to load the User FPGAs, and the other to reset all cores connected to the MPI network. The latter is controlled using GPIO from within the Control FPGA, and then propagated to the User FPGAs using one of the direct communication lines existent between them. A latency is associated to the propagation of the reset signal from the Control FPGA to the User FPGA. This latency is added to the profiling timestamps of the User FPGAs to assure tight synchronization between their clock cycle counters and the clock cycle 


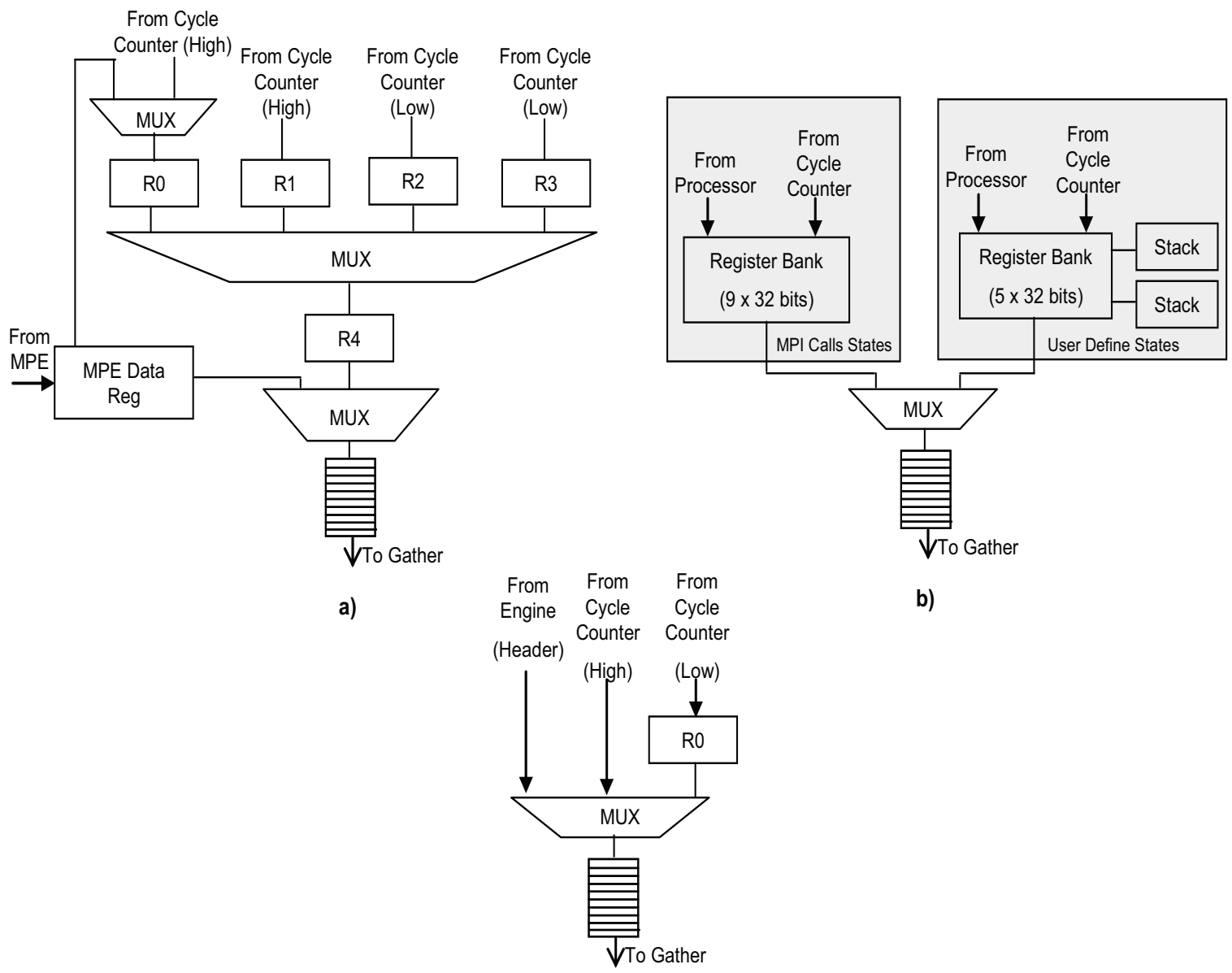

c)

Figure 3.3: a) Communication Tracer b) Computation Tracer (Processor) c) Computation Tracer (Engine)

counter of the Control FPGA.

FPGAs on the same board use the same crystals to generate their clocks. Therefore, there is no need to account for clock drifting. Synchronization between all boards is done by sending their Control FPGA clock cycle counter to the root board. Contrary to synchronization within the same board, different boards have different crystals to generate their clocks signals, so clock drifting may occur between boards. Maintaining synchronization requires a periodic communication from all boards with the root board.

The Collector is responsible for all synchronization messages transmitted between boards. Because a MPI rank is often used to calculate which data goes to which computation element, 
and increasing the total number of ranks may increase hardware overhead, collectors do not have a MPI rank associated to them. However, they still use the MPI infrastructure to send synchronization messages between boards by assuming the rank of a nearby computation element when injecting messages into the network. Similarly, the collectors monitor all messages sent to that rank and remove the synchronization ones, identified by a reserved tag. No computation element is ever aware of this process.

The timestamps are updated post-mortem. It's important to remove any communication overhead of the synchronization packages, so the time it took to deliver the messages is not introduced as clock drifting. The first time the synchronization occurs, the root board replies to all other boards so the round trip time can be calculated. This value is then used for all subsequent synchronizations. Because the round trip time can increase considerably if the synchronization message encounters traffic, synchronization messages are sent more often than needed, and ignored if the calculated drift is too high.

\subsection{Software}

The profiler library uses the same API as the MPI Parallel Environment of the MPICH distribution. Therefore, an instrumented source code, through embedded calls, can be easily ported from a traditional cluster to the TMD. However, some of the functions perform a different task or no task and exist only for portability reasons. MPE_Describe_state [16] is an example of such a function. Its task is to allow the user to define a state through two integers, a description and a color. To support this function it would be necessary for the computation elements to send these descriptions through the profiler network. This would create unnecessary traffic from a PowerPC or a Microblaze, and is impossible to support by a hardware engine that runs no source code. Therefore, all states are described in a configuration file, outside the TMD system. The system outputs the profiler logs in text format, which are then concatenated together. The amalgamated file is then sorted by time (from oldest to recent) and written in the CLOG2 
format used by the MPI Parallel Environment. The CLOG2 files will then be converted into SLOG2 (Scalable LOGfile), a format optimized for visualization, and used by Jumpshot 4 .

User states are created using embedded calls on the application code. States related with the TMD-MPI are already created within the TMD-MPI library and can be enabled through a flag at compilation time (only applicable if a soft-processor is not using TMD-MPE). 


\section{Chapter 4}

\section{Case Studies}

In this chapter, the functionality of the profiler is tested using four different case studies. The first two, Barrier and the heat equation, are simple applications included only to show how the profiler is able to demonstrate the communication patterns of a given application, running in software. The third case study, Linpack benchmark, is a demanding application both at the computation and communication levels used to rank supercomputers on the TOP500 list [18]. It also demonstrates the profiler being used with hardware engines. Finally, the last case study exposes a bottleneck that would pass unnoticible without a profiling tool.

\subsection{Barrier}

The barrier is a collective call in which no node will advance until all nodes have reached the barrier and it is used when the application requires synchronization points. This thesis demonstrates two versions: a sequential barrier and a binary tree barrier. For the sequential barrier the root node will wait for all other nodes to report to it and will then reply to all nodes so they can advance with the computation. This method scales very poorly as there will be a large amount of contention while the nodes communicate with the root node. For the binary tree version a node will report to its parent when its children finish reporting to it, assuring that when the root node gets a message, all other nodes have reached the barrier. The root node will then signal its 
children and so on until the signal reaches the leaf nodes. Fig. 4.1 shows communication patterns for the two barrier implementations. The numbers indicate the rank of the nodes. Fig. 4.2

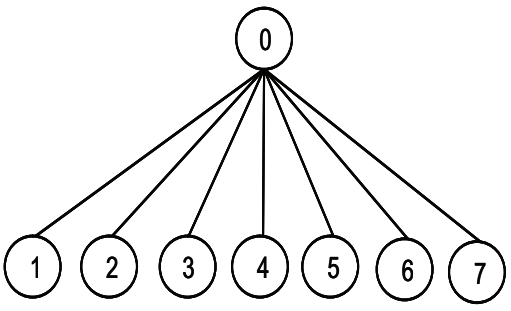

a)

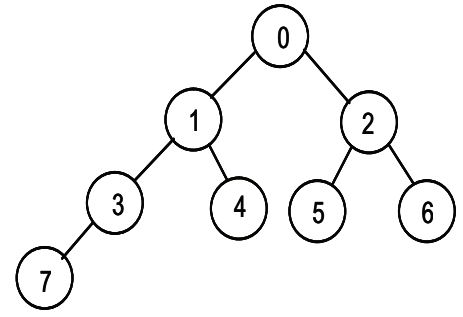

b)

Figure 4.1: Barrier Communication Patterns

and Fig. 4.3 show the data retrieved from the profiler when running the two algorithms on one BEE2 board with two PowerPCs in each User FPGA (eight-node network). The horizontal axis shows the time elapsed, and the vertical axis shows the node ranks. Each rank is represented by two timelines, one for receives (RECV), and another for sends (SEND). Because the Barrier only synchronizes the nodes and does not transfer any data, only the request to send packets and acknowledge packets can be seen on the figures. In the sequential algorithm, rank 0 can be seen receiving from all other ranks and then replying to each of them sequentially. During the execution, the non-root ranks are stalled, waiting for rank 0 as shown by the bars on the Receive timelines indicating the times when the nodes are waiting in the receive state. In the Binary Tree algorithm the leaf nodes $(4,5,6,7)$ can be seen starting the barrier by communicating with their respective parents. When rank 0 finishes receiving messages from both its children, it replies back to them and the tree is traversed downwards until it reaches all leaf nodes. Both while traversing the tree upwards and downwards, communications are done simultaneously between ranks. During the execution of the barrier, up to two Send-Receive pairs can be seen occurring at the same time. The binary tree algorithm scales logarithmically and does not introduce considerably more overhead then the sequential version, making it faster for three or more processors as expected. 


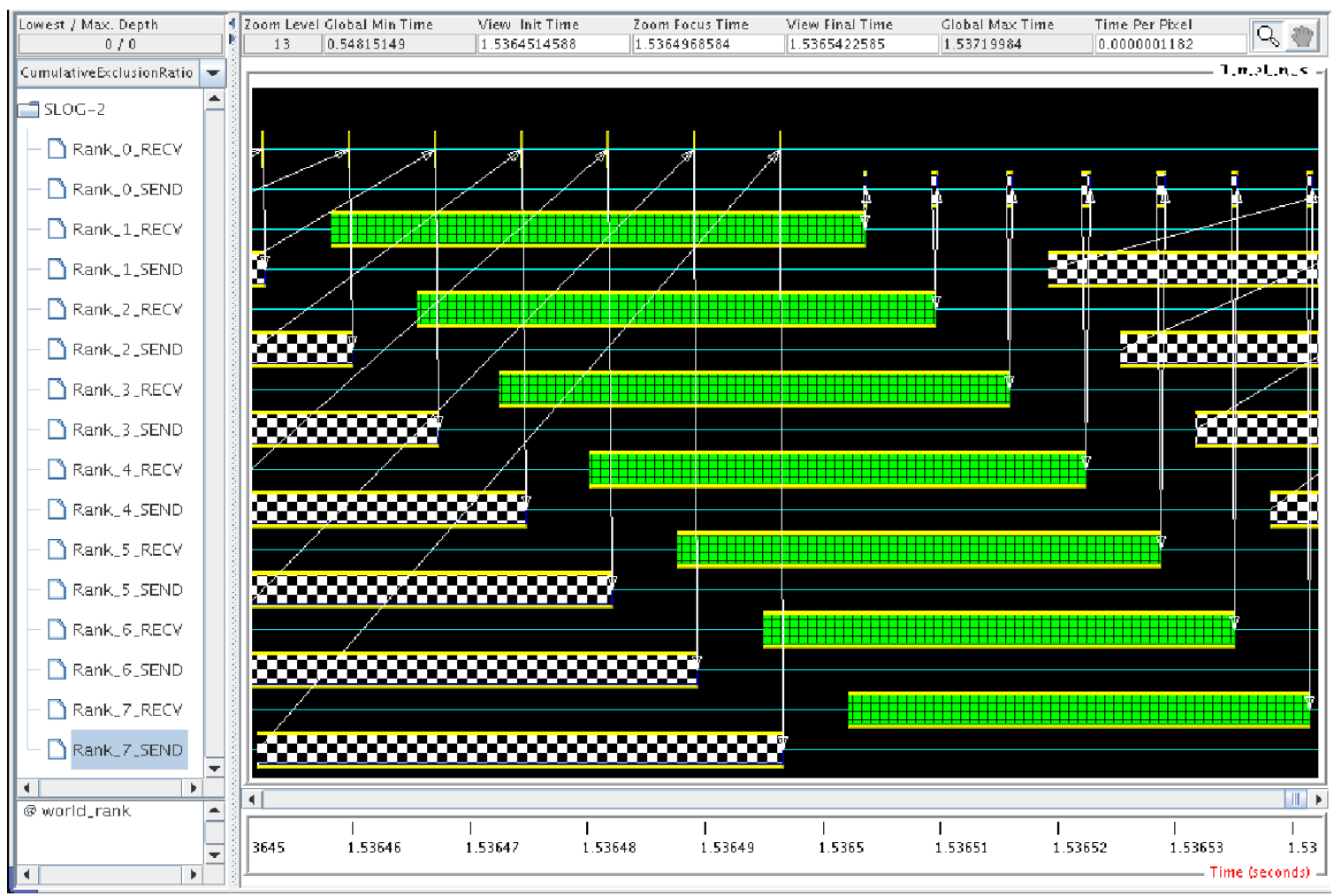

Figure 4.2: Barrier implemented sequentially

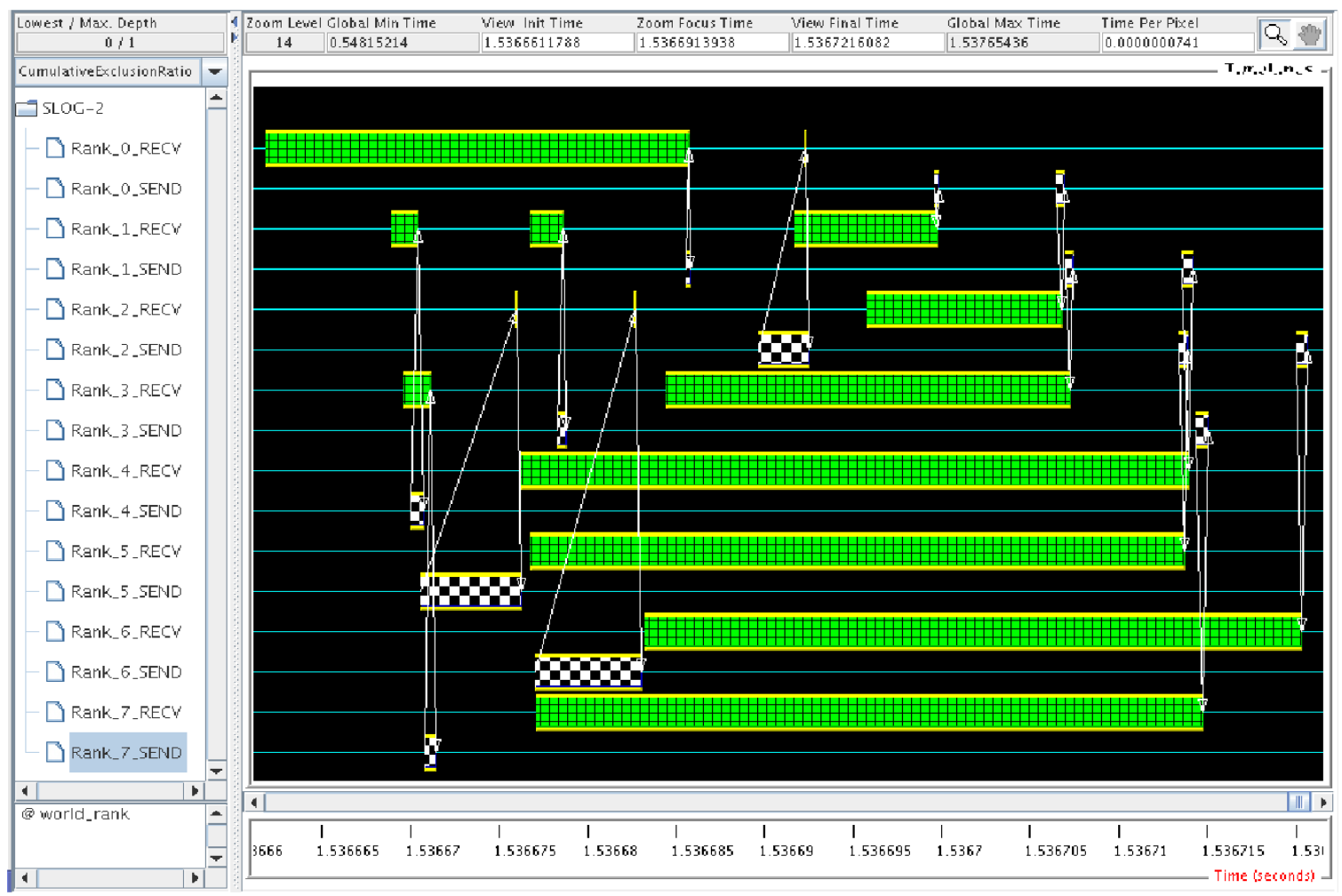

Figure 4.3: Barrier implemented as a binary tree 


\subsection{The Heat Equation Application}

The heat equation application is a partial differential equation that describes the temperature change over time, given an initial temperature distribution and boundary conditions. The thermal distribution is determined by the Laplace equation $\nabla(x, y)=0$ and can be solved by the Jacobi iterations method, which is a numerical method to solve a system of linear equations. The algorithm used in this paper was chosen for its simplicity and it is far from being the most optimized algorithm available. The goal of this section is to show the profiler features while comparing MPI blocking with MPI non-blocking calls, and not to obtain maximum performance.

The Jacobi iterations method is based on Equation 4.1, where $u$ is the matrix with the temperatures of the current iteration and $v$ the matrix with the temperatures of the previous iteration.

$$
v_{i, j}=\frac{u_{i-1, j}+u_{i+1, j}+u_{i, j-1}+u_{i, j+1}}{4}
$$

Traditionally the algorithm will iterate until the convergence criterion is satisfied:

$$
\sqrt{\sum\left(u_{i, j}-v_{i, j}\right)^{2}}<\epsilon
$$

For simplicity, convergence will be assumed after N_STEPS. The matrix is divided into equal parts between all nodes and the algorithm used consists of the following steps:

Step 1. Receive data from root node

Step 2. Exchange rows with neighbors

Step 3. Perform Computation

Step 4. If iteration number is less then N_STEPS go to Step 2 else go to Step 5

Step 5. Send data to root node 
Fig. 4.4 shows the data decomposition of the global matrix, and which rows are exchanged between neighbors. Only the first and last row of each of the smallest matrix depends on data from the neighbors matrices. Other algorithms exist that take into account extra levels of rows to calculate the new temperatures, but only a first order algorithm was implemented in this work.

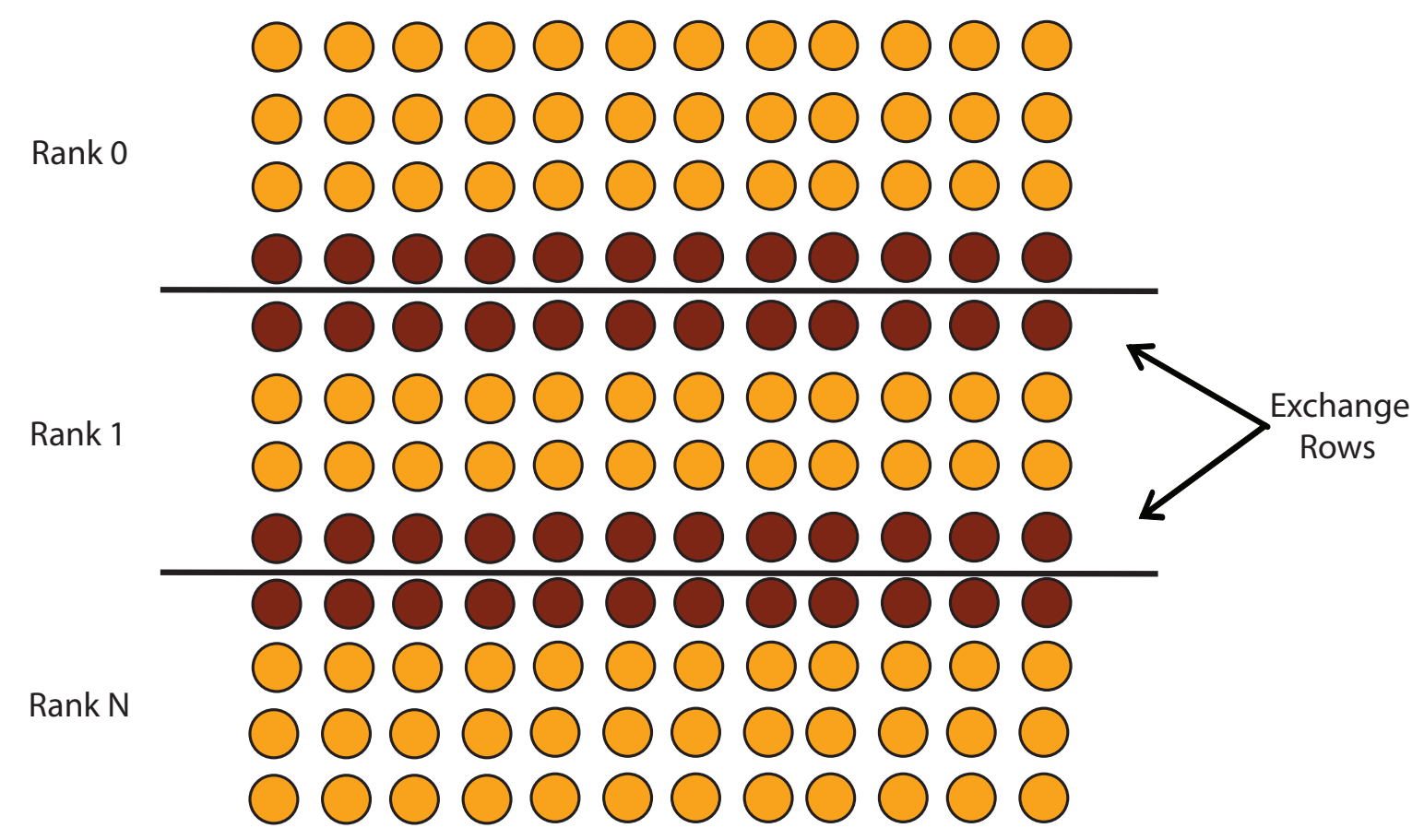

Figure 4.4: Data decomposition and point-to-point communication in Jacobi algorithm

MPI supports both blocking and non-blocking calls. Non-blocking calls can be extremely useful when there is computation that carries no data dependence with the data to be transferred. If the system architecture allows, the computation can then be done simultaneously with the data transfer. However, depending on the implementation, non-blocking calls can bring extra overhead and care is needed to decide when it is advantageous to use them over the blocking calls. Parallel systems like the TMD can take full advantage of such calls. Each node can have a TMD-MPE core that will be in charge of the communications, freeing the CE to perform whatever computation there may be.

We implemented two different versions for the calculation of the heat equation that differ only on the type of communication used in Step 2. When using blocking calls, the nodes will 
stall computation while rows are exchanged between neighbours, regardless of having independent rows that can be calculated while waiting for the row exchange. The non-blocking calls on the other hand, will allow computation of all independent rows on each node after initiating the row transfers, improving the computation to communication ratio. Both versions of the application were run with a matrix of 32 by 512, with N_STEPS $=64$, on one BEE2 board with two PowerPCs in each User FPGA. The profiler log for the version using the blocking calls can be seen in Fig. 4.5, and using the non-blocking calls in Fig. 4.6. The hardware configuration is the same as the previous case study. However, for this example we also instrumented the source code to visualize the processor computations. Therefore, each rank is now represented by three timelines, receives (RECV), computation (COMP) and sends (SEND). The profiler $\log$ visualization of the blocking version shows a clear break of computation while the rows are exchanged between processors. Although the program is being run in parallel across multiple nodes, each node is running sequentially, such that receives, sends and computation never overlap. The non-blocking version, on the other hand, takes full advantage of the communication infrastructure, and while the TMD-MPE performs the communications, the PowerPC is computing all independent data. This can be seen on the profiler log visualization of the non blocking version, where the majority of the ranks are receiving, sending and computing simultaneously. 


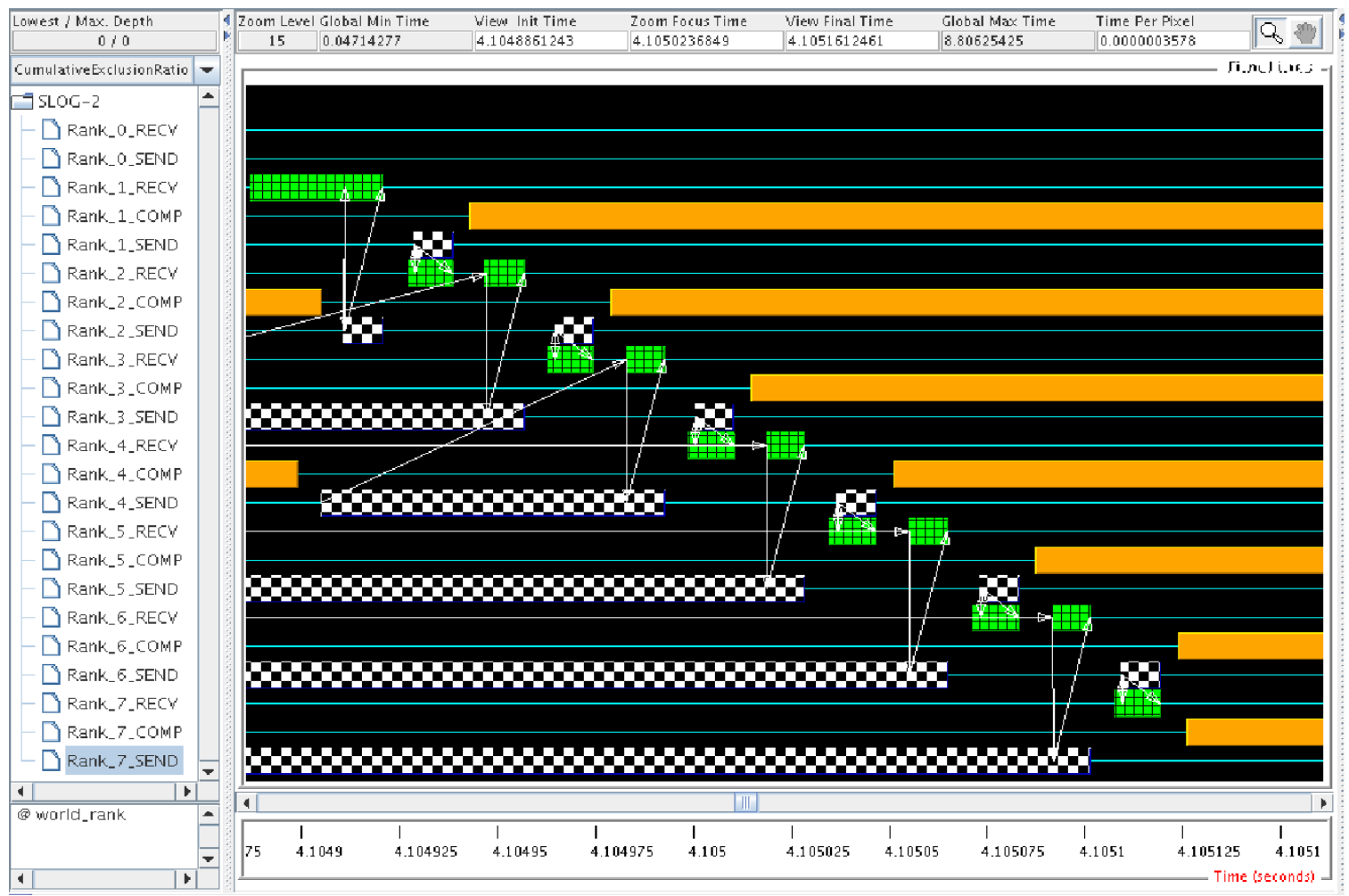

Figure 4.5: Using blocking calls

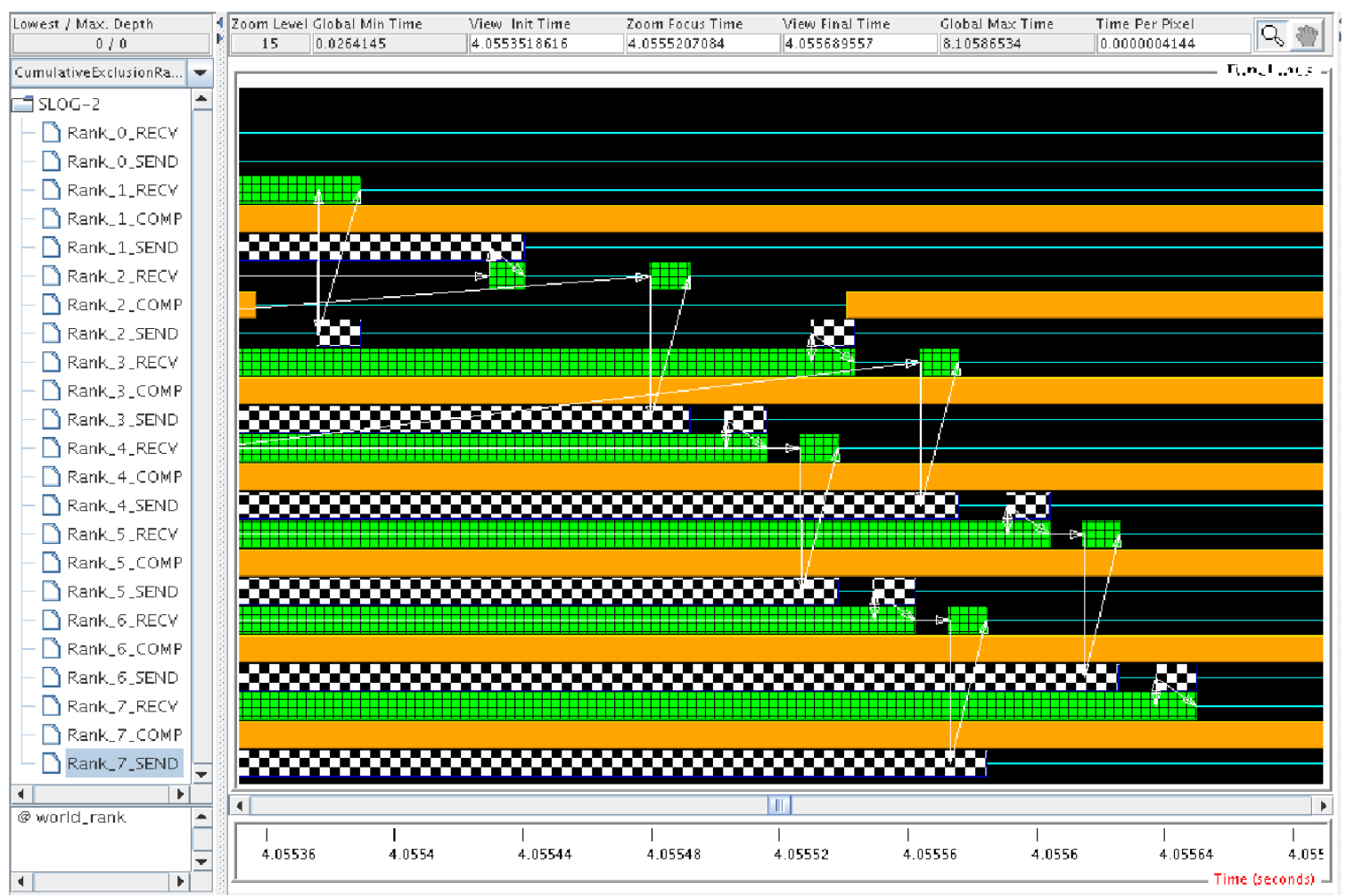

Figure 4.6: Using non-blocking calls 


\subsection{The LINPACK Benchmark}

The LINPACK benchmark [19] consists of subroutines used to analyze and solve systems of linear equations. Such systems are solved through matrices calculated by using matrix decomposition.

LINPACK was originally written in FORTRAN and used to solve systems of linear equation of size 100 by 100. In 1979, the LINPACK User's Guide [20] included the performance data gathered from the most widely used computers of the time. Since then, LINPACK as been evolving to adapt to the ever changing reality of computer performance. The default matrix size was increased to 1000 by 1000 , reflecting the increase of computer performance. Also, with the increase of processor frequencies without a comparable increase of memory frequencies, memories became more and more a bottleneck. Hierarchical memory structures, where closer memories to the processor are faster, were design to shorten this gap. However, the closer the memory is to the processor, the smaller it is. If data cannot be found in the highest hierarchical level memory (cache miss), the application can suffer penalties on the order of hundreds or thousands of cycles. To prevent this, memory management policies can take advantage of the concept of locality of reference, where, if a given location in memory is being referenced, then chances are that memory locations around it my also be referenced (Spatial Locality), or, that memory space may be referenced again in the near future (Temporal Locality). LINPACK, initially implemented using LEVEL 1 BLAS [21] subroutines that do all calculations as vector-vector, was subsequently implemented using LEVEL 2 BLAS and LEVEL 3 BLAS subroutines that do vector-matrix and matrix-matrix calculations respectively. This allows maximization of data reuse by fetching blocks of data containing those matrices.

$$
A x=b
$$

Equation 4.3 describes the linear system to be solved. The first step is to perform an $L U$ decomposition with partial pivoting of matrix $A$ obtaining: 


$$
P A=L U \Rightarrow L U x=P b
$$

where $P$ is a permutation matrix used to exchange the rows of matrix $A, L$ is the lower triangular matrix and $U$ the upper triangular matrix of $A$. The system is then solved by first substituting $U x$ by $y$ and calculating $y$ as follows:

$$
L y=P b
$$

and then using $y$ to calculate $x$ as shown in Equation 4.6.

$$
U x=y
$$

Finally, a residual value can be calculated in order to verify the accuracy of the result. The residual value is calculated by:

$$
\begin{gathered}
r=\|A x-b\| \\
r_{n o r m}=\frac{\|A x-b\|}{n \cdot\|A\| \cdot\|x\| \cdot \epsilon}
\end{gathered}
$$

where $n$ is the size of the matrix and $\epsilon$ the relative machine precision. If the $r_{n o r m}$, the normalized residual, is of order $O(1)$ the result is considered accurate, and if greater than order $O(100)$, the result is considered incorrect.

Fig. 4.7 shows the FPGA configuration used for this application. Each User FPGA contains two specialized hardware engines [22] that perform the $L U$ decomposition. The User FPGA 1 of the first board also contains a PowerPC that solves the linear system after the decomposition is done and calculates the residual value. The objective of this section is to profile the decomposition, therefore, the PowerPC is not being profiled. Each TMD-MPE is connected to two Tracers (receives and sends), and each engine is connected to one Tracer that profiles the 


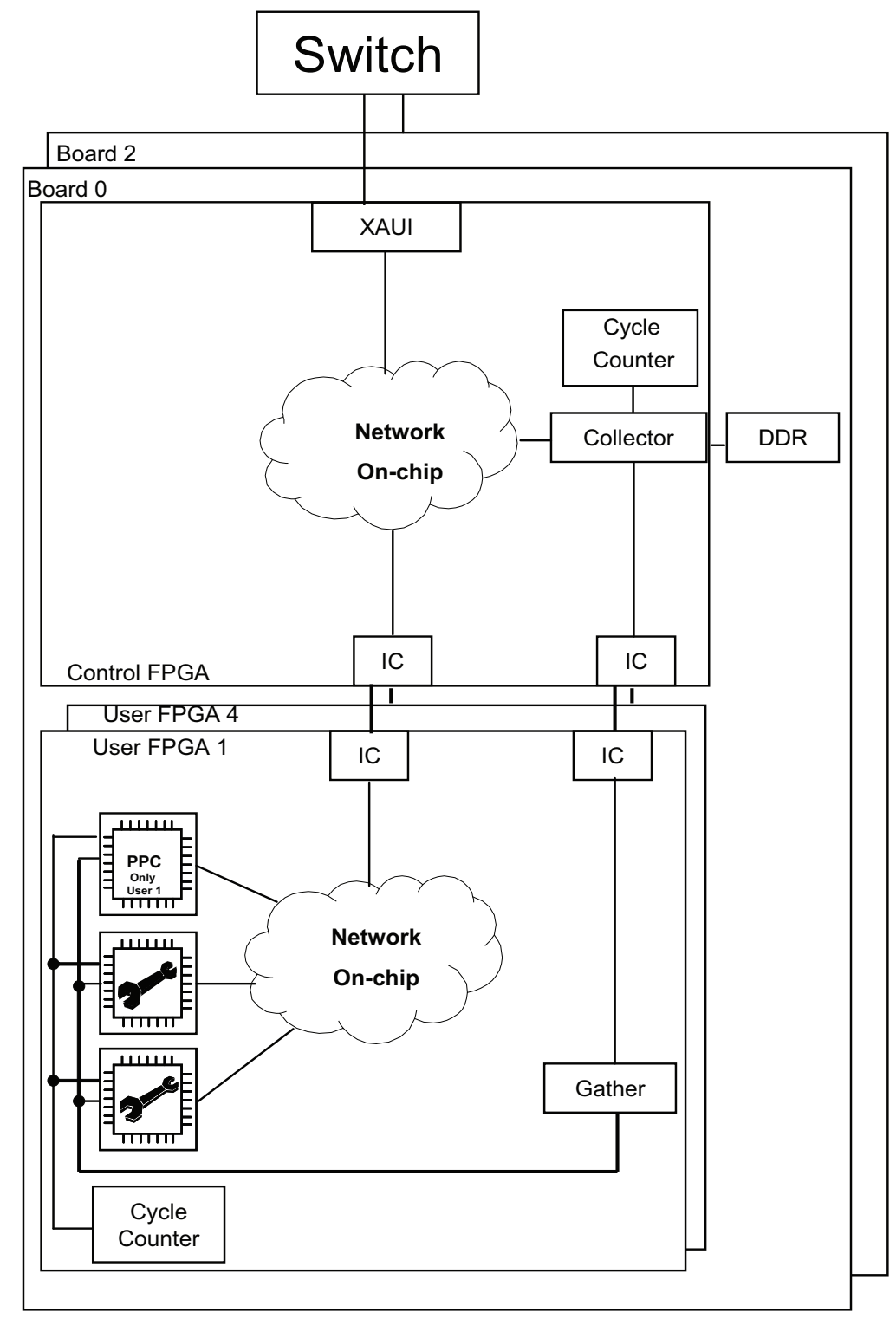

Figure 4.7: Linpack Benchmark Testbed

engine's computation. The Control FPGA contains no computing element, and is used to route communications off-board and to synchronize the clock counter between boards. In total, the application was run with seventeen ranks (two BEE2 boards). The matrix being solved is of size 200x200 and columns are cyclically distributed between ranks (with the exception of the PowerPC).

Fig. 4.8 shows an iteration of the Linpack Benchmark. First, rank 1 computes the pivot and scales the column. Then it broadcasts the column to all other engines so their columns can be 
Table 4.1: State's Duration

\begin{tabular}{|c|c|c|c|}
\hline RANK & MPI_Recv & MPI_Send & Computation \\
\hline Engines & $67 \%$ & $6.5 \%$ & $23.6 \%$ \\
\hline
\end{tabular}

updated. Looking at Fig. 4.8, rank 1 spends most of the time sending to other ranks, meaning that the Broadcast is taking longer then the computation itself. Because of that, all ranks besides the last four idle considerably waiting for rank 1 to finish its computation. A larger matrix would reduce this problem by increasing the computation state. However, because the application has to be prepared for all sizes of matrices, a solution for this delay would be to implement the Broadcast in a similar fashion as the Barrier in Section 4.1. This would reduce the Broadcast length and consequently the iteration length by more then half. Another noticeable delay can be seen between the starting point of the computation of rank 8 and rank 9, which is double the delay between rank 7 and rank 8 . This delay can be attributed to the off-chip communications through the XAUI interface. Rank 9 is the first rank on the second BEE2 board.

Fig. 4.9 shows a general view (32 iterations) of the Linpack Benchmark. The cyclic distribution of columns between ranks can be seen by the constant exchange of which rank is doing the Broadcast. Table 4.1 shows the average percentage of time each state is active. 


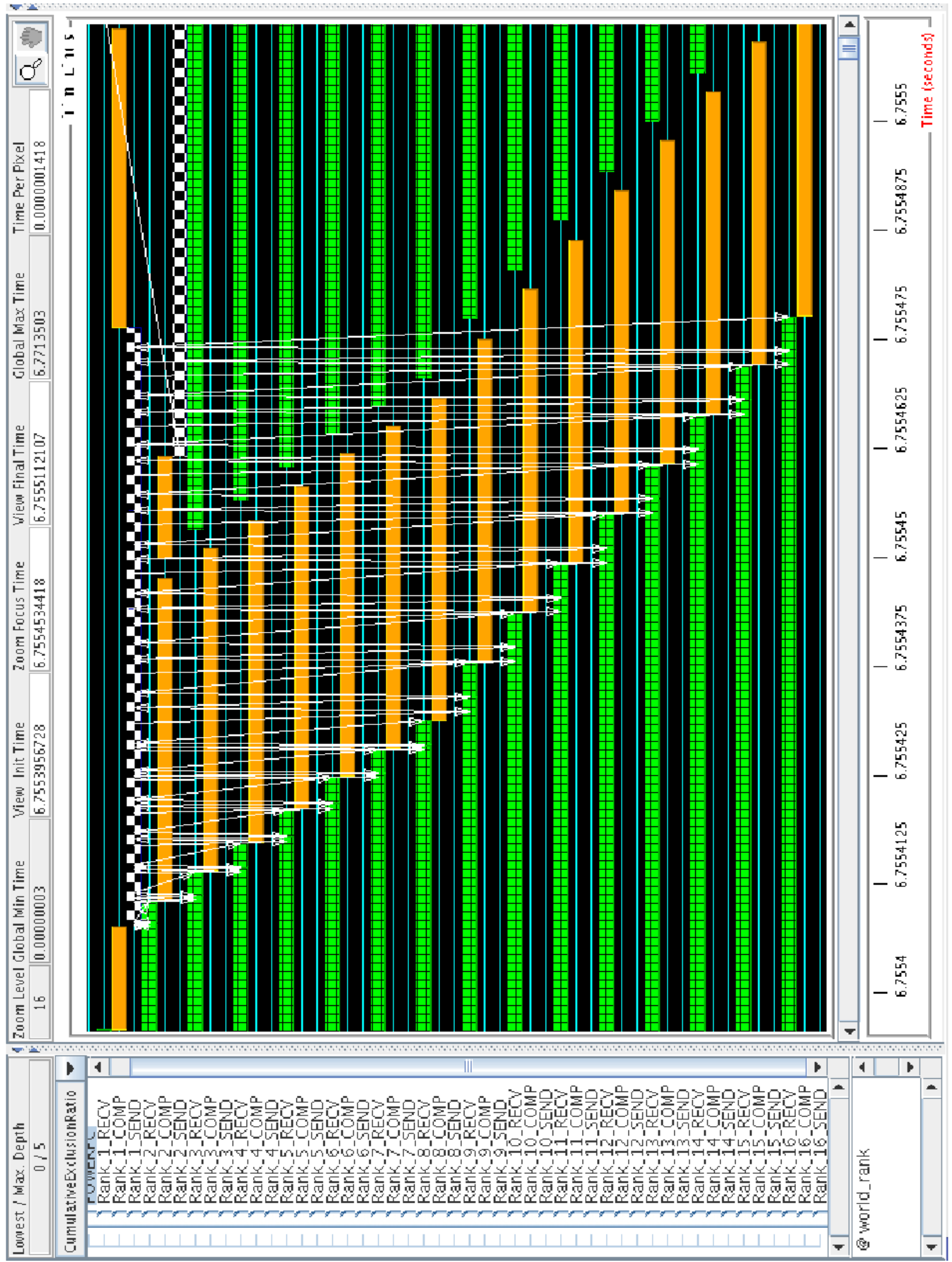

Figure 4.8: Linpack Benchmark Iteration 


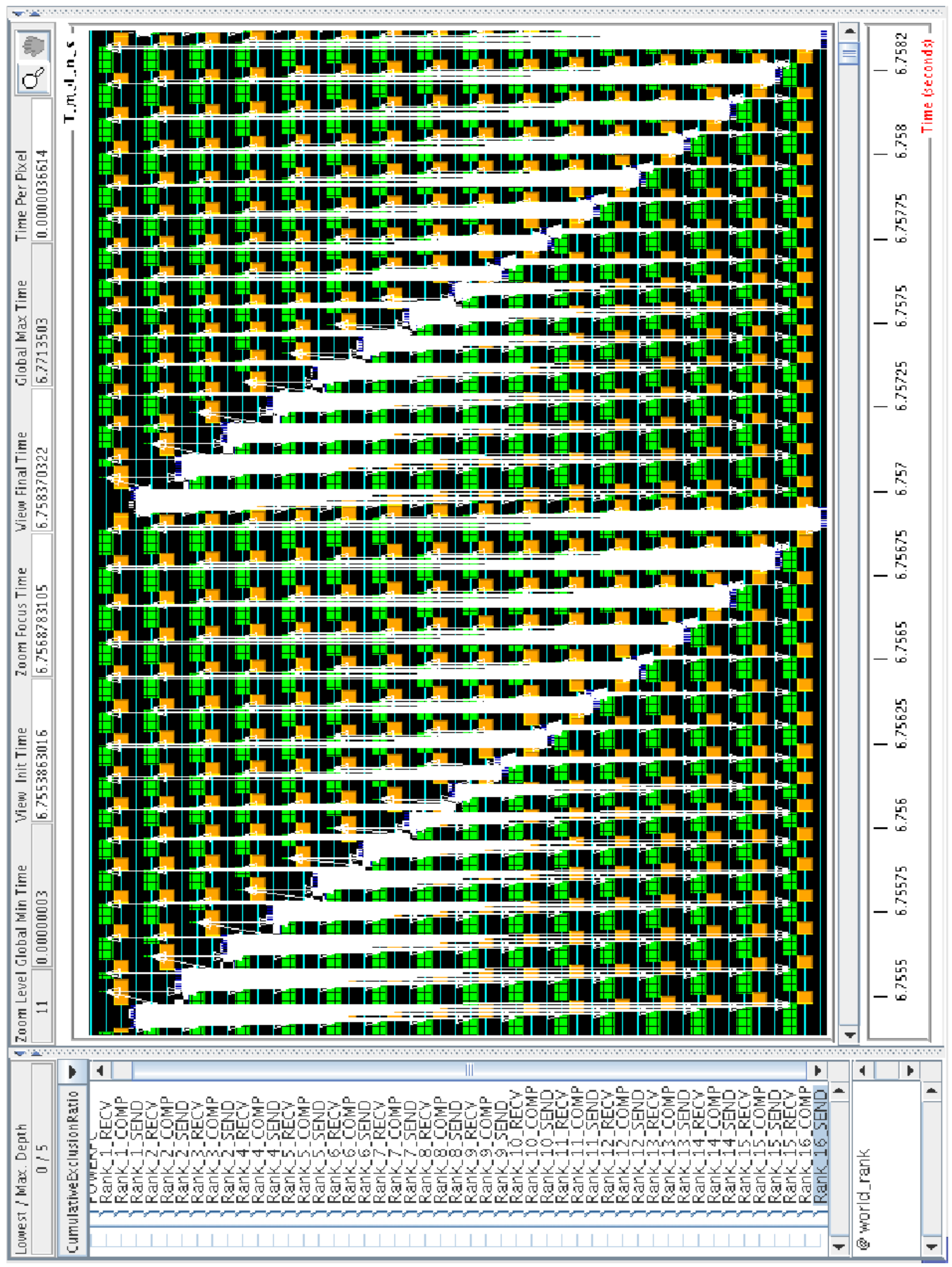

Figure 4.9: Linpack Benchmark General View 


\subsection{TMD-MPE - Unexpected Messages Queue}

When a computing element uses the TMD-MPE, almost all communication overhead of sending/receiving messages is transfered to the TMD-MPE. Independent of using blocking or nonblocking calls, a message is only sent by a rank after it receives the acknowledge of the other rank. If a request is made while the receiving rank is occupied with a transaction, this request needs to be recorded so it can then be replied to when its turn comes. These requests are saved in a queue called the Unexpected Messages Queue. When a computing element issues a receive, the TMD-MPE will first check its Unexpected Messages Queue for matches. If a match is found, that entry is taken out of the queue, the queue is reorganized and the TMD-MPE will process the receive.

Running the system in Section 4.1, turned attention to this queue. Fig. 4.10 shows the exact same application as Fig. 4.2. However, instead of giving a general view of the barrier, the image is zoomed in to give prominence to when the root rank receives from one of the other ranks. Also, a computation tracer is connected to the TMD-MPE so the time it spends searching the Unexpected Messages Queue is visible with Jumpshot4.

Intuitively, it would be expected that the closer a rank is to the root rank, the faster this transaction will be, since less hops are necessary to reach the root rank. However, the exact opposite occurs, the fastest transaction is when the root rank receives from the farthest rank. This is caused by the time spent searching the Unexpected Messages Queue, as shown in Fig. 4.10 (solid grey state inside rank 0 receives).

The application used in Section 4.1 consists of barriers being executed one after the other. On the sequential version, as soon as the root rank issues a send to another rank, giving it permission to advance in computation, this rank will immediately start the next barrier and send a request to send message to the root rank. Because the root rank is busy with other ranks, this request to send message will be saved in the Unexpected Messages Queue. When the root rank starts the new barrier, it will gradually take all messages out of the Unexpected Message Queue.The original implementation of the TMD-MPE reorganizes the Unexpected Message 
Quеие by copying all entries over the removed entry one position down. Since the root rank only starts freeing ranks out of the barrier when all ranks report to it, the Unexpected Message Qиеие will become smaller as the barrier is executed, and its reorganization faster.

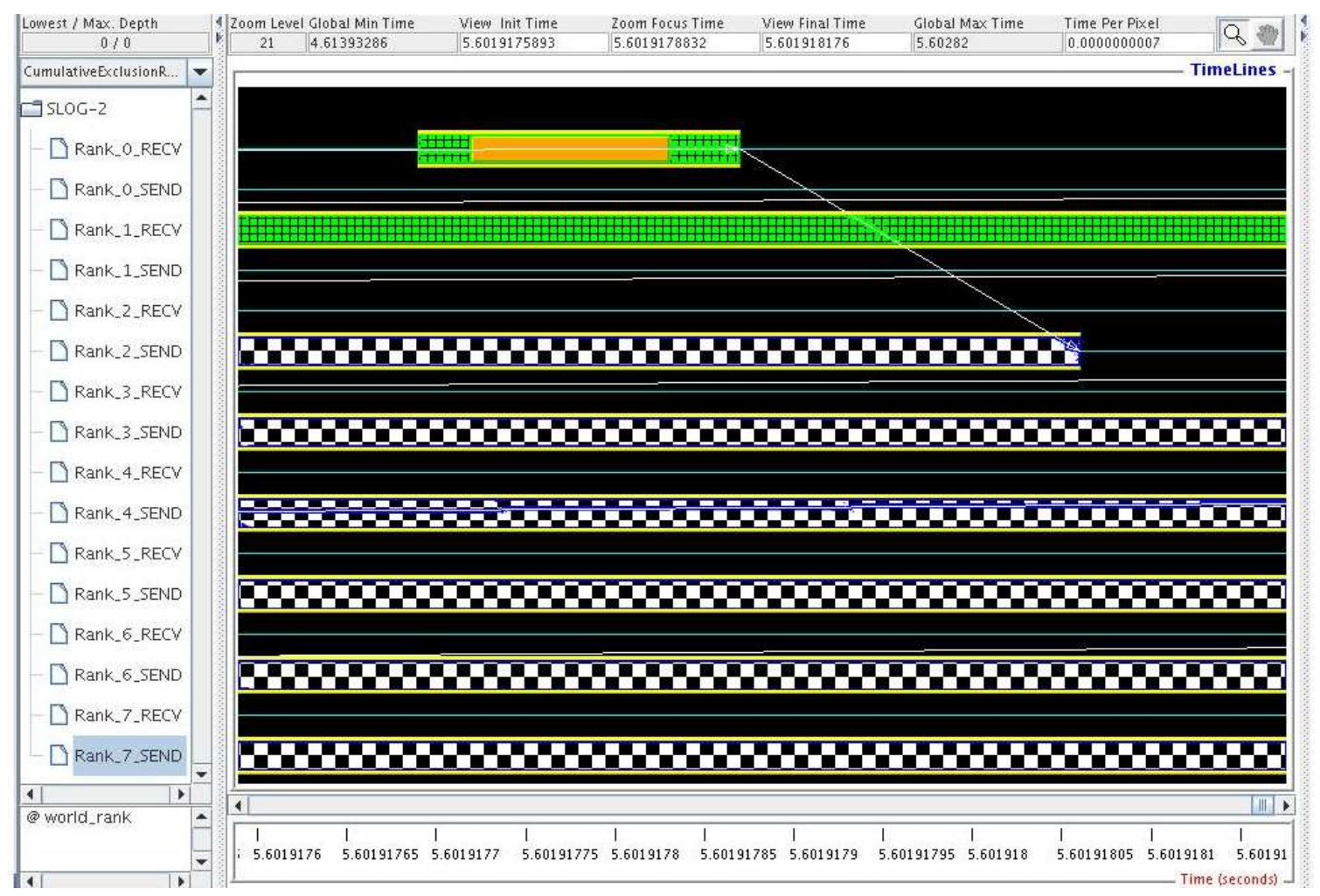

Figure 4.10: Barrier implemented sequential with TMD-MPE profiled

A better reorganization algorithm could reduce or avoid the penalty of having a long Unexpected Messages Queue. Instead of always starting the search from the same address in memory, two separate memory addresses can be kept: one where data starts, and the other were data ends. If the first element is removed, the starting address can be incremented, if the last element is removed, the ending address can be decremented. If the element is neither in the beginning or end of the queue, it could be interchanged with one of the edge elements and then removed. This algorithm would end the duration differences of the receives of Fig. 4.2. A different approach would be to take advantage of the communications being synchronous. That is, everytime a request to send is sent by a rank, that rank will not start any other transaction until the one it started is finished, limiting the number of on-the-fly messages to one per rank. 
If only one request package exists, then all of them can be kept inside the Unexpected Messages Quеиe indexed by the rank number and associated to a flag that would validate or not the content of that index. Searching and removing the element would take one cycle, decreasing considerably the time each one of those receives take. 


\section{Chapter 5}

\section{Profiler Overhead}

All applications, with the exception of the Linpack benchmark, were executed with the same hardware configuration. While the hardware overhead introduced by the profiler for the Control FPGA is constant throughout this work, on the User FPGAs the difference arises from the Tracers used to profile the PowerPCs, and the Tracers used to profile the hardware engines.

Table 5.1 shows the resource overhead of using the profiler for all configurations where the percentages in brackets are fractions of a Virtex II Pro 70 FPGA. Part of this overhead is due to the Tracer's buffers on the User FPGA and the collector's buffer on the Control FPGA. The size of these buffers can be reduced to save resources, however, profiling events may be lost, compromising part of the profiling data. Another option would be to use BRAMs for buffering, reducing the size of the cores. Unfortunately, BRAMs can be a scarce resource depending on the design, so we chose not to use them on the profiler. More specifically, each communication Tracer occupies 526 LUTs and 1000 flip flops. A Tracer for a processor will occupy 1196 LUTs and 1521 flip flops when profiling both communications and computation (no TMDMPE), and 855 LUTs and 1200 flip flops when profiling just computation. The overhead of a computation Tracer for a hardware engine will depend on the hardware engine itself because each event being captured may require some custom logic to make the event available to the Tracers. However, the one used for this work is capable of fulfilling the profiling needs of most 
Table 5.1: Tracers Overhead

\begin{tabular}{|c|c|c|c|}
\hline FPGA & LUTS & Flip Flops & BRAMs \\
\hline Control & $3856(5 \%)$ & $1279(1 \%)$ & $0(0 \%)$ \\
\hline User & $4079(6.1 \%)$ & $6630(10 \%)$ & $0(0 \%)$ \\
\hline User Linpack & $3285(4.9 \%)$ & $5366(8.1 \%)$ & $0(0 \%)$ \\
\hline
\end{tabular}

engines. This particular computation Tracer will occupy 396 LUTs and 701 flip flops.

The overhead introduced by the software libraries to profile the source code of the Microblaze or the PowerPC consists of communications with an FSL and GPIO, which on average will take one hundred and fifty cycles per event. As long as the programmer takes some care on choosing the length of the user states, this will not affect performance considerably. If the communications are being profiled through software, meaning the processors do not have the aid of a TMD-MPE unit, the system will suffer a performance decline, making the data retrieved from the profiler less helpful to improve performance. However, the data can still be used to study the application's communication patterns, and when running a system to obtain maximum performance, all processors will have the aid of a TMD-MPE unit. Profiling the TMD-MPE or any hardware engine will not affect system performance as long as the system frequency is maintained. This may not be possible when the FPGAs are close to full utilization when it becomes more difficult to meet timing during the placement and routing phase of the FPGA tools. 


\section{Chapter 6}

\section{Profiler Implementation on Other}

\section{Platforms}

\subsection{Backplane}

The Backplane consists of five Amirix AP1100 PCI development boards. Each board has a Xilinx 2VP100 FPGA with 128 MB of DDR RAM, 4 MB of SRAM, support for high-speed serial communication channels, two RS232 serial ports, and an Ethernet connector. Each FPGA board is connected to a 16-slot PCI backplane, which provides power to the FPGA boards. In addition to the FPGA boards, there is a Pentium 4 card plugged into the backplane used only for the FPGA configuration and to provide access to I/O peripherals, such as keyboard, mouse, monitor, and a hard disk drive. The operating system running on the Pentium 4 card is Linux. The boards are interconnected by a fully-connected topology of Multi-Gigabit Transceiver links (MGTs), which are high-speed serial links. Each MGT channel is configured to achieve a full-duplex bandwidth up to 2.5 Gbps. Fig. 6.1 shows an image of the Backplane.

Unlike the BEE2 board, each board on the Backplane only contains one FPGA. Therefore, each FPGA is responsible for either saving or sending all profiling data generated within it. While the BEE2 board contains 20 GB of DDR RAM, 4 GB per each FPGA, the Amirix board 


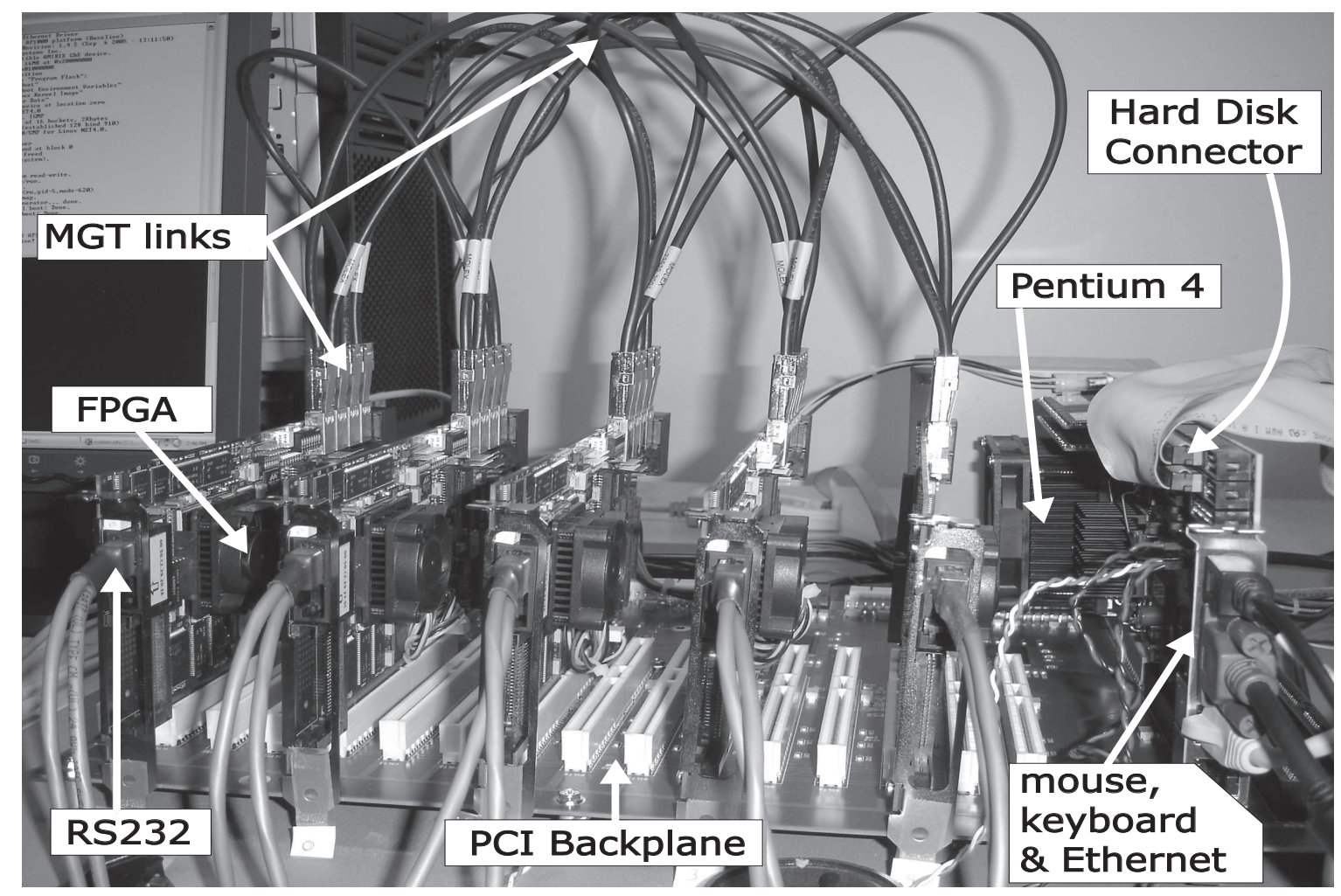

Figure 6.1: The Backplane

contains only $128 \mathrm{MB}$ of DDR RAM. Whereas a $128 \mathrm{MB}$ of memory is capable of saving over 3,500,000 profiler packages, allowing the profiling of an application for a considerable time, problems may arise when the DDR memory is being used by the application, which is the most common case considering the memory limitations of an FPGA and the lack of memory options of this particular board. Therefore, the best solution is to send all profiling data outside the board where enough memory exists. This can be achieved by using either the ethernet or by using the MGTs. The former provides a typically not critical connection for the application, if used by it at all. Also, $100 \mathrm{Mbits} / \mathrm{s}$ is more then sufficient to send all profiling data while reducing the amount of buffering needed. The latter, uses the resources already in place for the application. However, to avoid delays created by the profiling packets, priority should be given to the application packets traveling through the network, implying the need to have larger buffers that can keep the profiling packages while the network is being used by the application. Both cases can connect directly to a typical PC not used by the application that 
caries has memory to hold all profiling data of all FPGAs. Fig. 6.2 shows the diagram for both implementations.

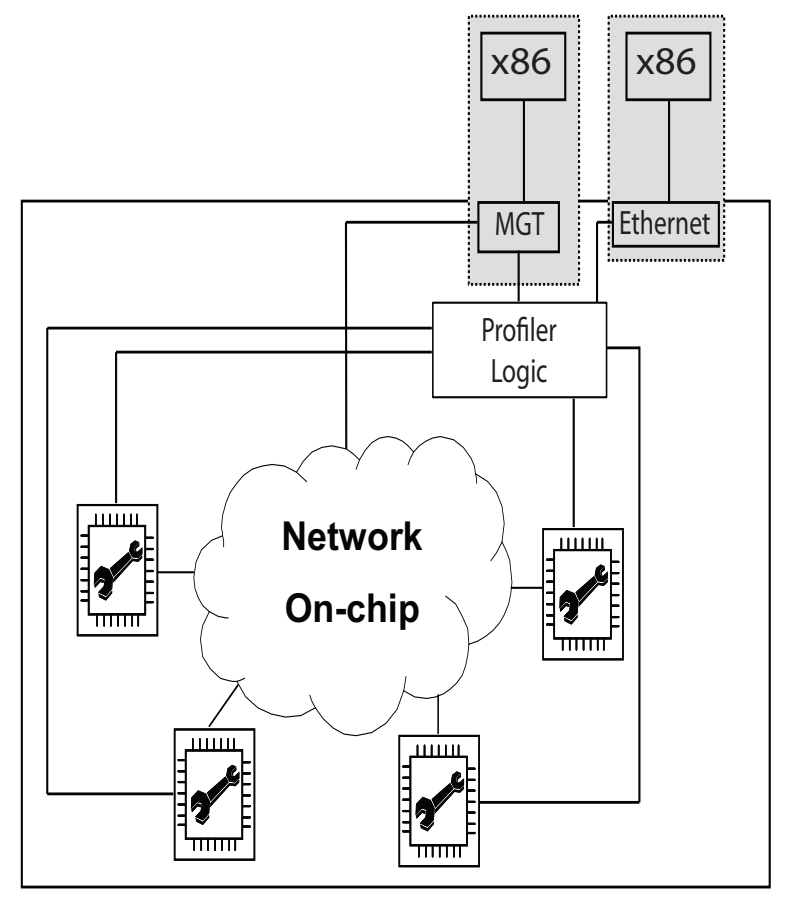

Figure 6.2: Possible Implementation of the Profiler on the Backplane

\subsection{HPRC Implementation Platform}

This system consists of an Intel 4-Processor Server System S7000FC4UR. One of the sockets has a Xilinx Accelerated Computing Platform (ACP) consisting of a stack of PCB modules. The first layer contains the bridge FPGA (XC5VLX110) and is physically placed in one of the X86 sockets on the motherboard. It provides a connection point to Intel's Front Side Bus (FSB) and provides communications for the upper-level layers. The second layer contains two FPGAs (XC5VLX330) used for computation in the same PCB. All FPGAs are directly connected by LVDS lines in the same layer and between layers. Another socket has an Intel Quadcore Xeon processor. The Xilinx ACP platform and the X86s share 8 GB of RAM through the FSB, and the machine is running a standard SMP Linux distribution. Implementing the profiler in this platform is in part similar to Section 6.1. In both cases, the profiling packages need to be sent 
out of the FPGA. Since a large memory is available on the FSB bus, the profiler can connect to the MPI Shared Memory Bridge and as with the last example, when no data is being sent out the FPGA, the profiling data can be copied into the shared memory. After the MPI_Finalize, one of the $x 86$ processors can use the profiling data to create a log file of the application execution. Fig. 6.3 shows the diagram of the implementation.

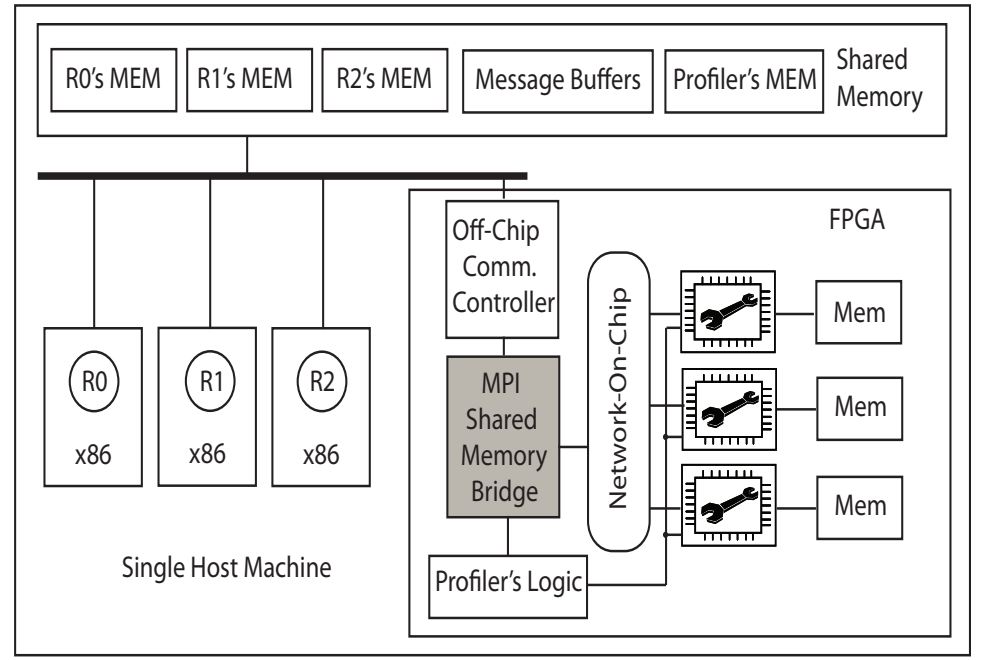

Figure 6.3: Possible Implementation of the Profiler on the HPRC Platform 


\section{Chapter 7}

\section{Conclusions}

To obtain full performance on FPGA systems, it is necessary to have a complete understanding of the application to avoid unexpected behavior that may undermine performance. A tool that allows the user to visualize what each core is executing at a given moment can bring the insight needed to avoid possible bottlenecks. In this work, we present a profiler that not only allows that knowledge to be retrieved from the system, but does so without affecting the performance of the system significantly. All major features of the profiling software environment provided by MPICH were implemented on a FPGA system.

First, the profiler is used in two simple case studies: a barrier and a heat equation application using the Jacobi iterations method. In both of them we are able to demonstrate the communication patterns and can use that knowledge to improve the application either by changing the algorithm to a more scalable one or by using more suitable communications calls so all levels of parallelism provided by the hardware can be used. Also, a bottleneck was found and corrected on the TMD-MPE that, without the profiler, would most likely pass unnoticed. Finally, the profiler is used to study the behavior of the Linpack benchmark, and is able to point to bottlenecks that reduce performance considerably.

The reconfigurability of FPGAs allows us to instantiate only the necessary hardware for performance analysis, minimizing the hardware overhead. Moreover, that hardware runs par- 
allel with the main application, and becomes intrusive only when communication resources are shared, or when the system frequency is not mantained. Performance analysis on reconfigurable hardware also increases the number of events available to the user, since unlike software profiling, we're not limited to events related with a processor. Our scope is now the full hardware system and we can accurately profile network components in addition to CEs. Compatibility with an existing profiling software environment reduces the learning curve of this tool and allows comparisons between traditional clusters and reconfigurable systems-on-chip.

This type of performance analysis (post-mortem) works well for applications with constant behavior throughout their execution. The profiler is able to aid the designer achieve a better network topology, better algorithms and even improve some hardware engines. For an application which behavior varies, the profiler can aid with building a system based on initial conditions. Overall, it provides a good starting point for performance analysis on the TMD system. 


\section{Chapter 8}

\section{Future Work}

With this working profiler the next step will be to focus on other computing demanding applications besides the Linpack benchmark that contain more complex communication patterns (e.g. molecular dynamics). With the increase in complexity of the applications, a tool that can perform a pre-analysis of the data and identify possible bottleneck areas can become extremely useful.

Improvements on the hardware blocks to reduce their footprint and increase functionality, such as track critical messages, are also scheduled.

Microblaze and PowerPC computation is being profiled intrusively through an FSL and GPIO connection. To avoid this penalty, the Tracer can profiler computation by accessing the program counter of each of the processors using a similar method as SnoopP or Xilinx Microblaze Trace Core (XMTC) [13].

Porting the profiler into the HPRC implementation presented in Chapter 6 will also be done in the near future.

At the moment, all analysis is being done post-mortem. Another option would be to implement a profiler that would be more restricted in the amount and type of data that it could retrieve, allowing real-time analysis for systems with dynamic behavior. Such profiler could work in conjunction with the one presented in this paper, where one would be used to optimize 
the initial configuration of the system, and the other to adapt the system to dynamic behavior. 


\section{Chapter 9}

\section{Appendixes}

\subsection{Appendix A}

In this appendix, an example system is provided and the interface of each hardware block is explained.

\subsubsection{Example System}

Fig. 9.1 shows the connections between different blocks of the profiler. This example considers only one engine being profiled in the User FPGA. Both communication and computation are being profiled. Fig. 9.1 can be use as reference for Section. 9.1.2, Section. 9.1.3 and Section. 9.1.4 


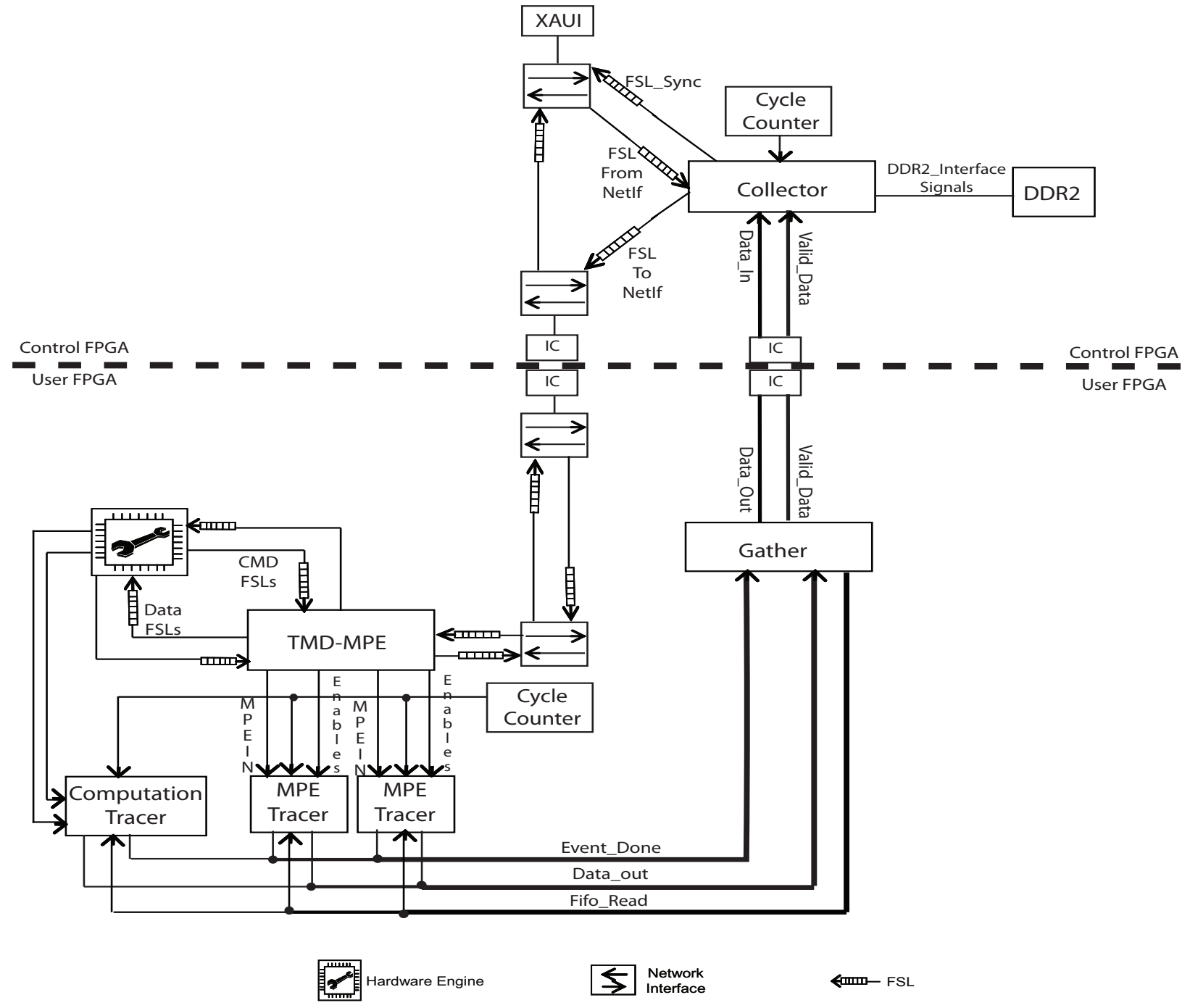

Figure 9.1: Example system 
Table 9.1: MPE_Tracer's Interface

\begin{tabular}{|c|c|c|}
\hline Signal Name & MSB:LSB & I/O \\
\hline Clk & & I \\
\hline Clk_Gather & & I \\
\hline Rst & & I \\
\hline Cycle_Counter & $63: 0$ & I \\
\hline MPE_In & $128: 0$ & I \\
\hline Enables & $1: 0$ & I \\
\hline FIFO_Read & & I \\
\hline Event_Done & & O \\
\hline Data_Out & $32: 0$ & O \\
\hline
\end{tabular}

\subsubsection{Tracer}

Regardless which type of Tracer block is being used, all Tracers have the same outputs and interact with the Gather block the same way. In this section, the MPE_Tracer is used as an example.

The Tracers operate at the same frequency as the computing element connected to them, which may be different than the frequency of the Gather block, hence two clock inputs are required as shown in Table. 9.1. The input Cycle_Counter comes from the Cycle_Counter block (shown in Fig.9.1 and it's used to obtain the timestamps for each profiler packet. The inputs MPE_In and Enables come both from the TMD_MPE and are used to receive data from the TMD_MPE and to enable that data, respectively. Input Fifo_Read is issued by the Gather, whenever there are profiling packets awaiting to be sent on the Tracer, and the Gather is prepared to receive them. The output Event_Done is used to signal the Gather when a profiling packet becomes available. Output Data_Out is used to send the profiling packets to the Gather block. 
Table 9.2: Gather Interface

\begin{tabular}{|c|c|c|}
\hline Signal Name & MSB:LSB & I/O \\
\hline Clk & & $\mathrm{I}$ \\
\hline Rst & & $\mathrm{I}$ \\
\hline Data_In & $\left(33^{*}\right.$ N_PROFILERS-1:0 & $\mathrm{I}$ \\
\hline Event_Done & N_PROFILERS-1:0 & $\mathrm{I}$ \\
\hline FIFO_Read_Tracers & N_PROFILERS-1:0 & $\mathrm{O}$ \\
\hline Sending_Data & & $\mathrm{O}$ \\
\hline Data_Out & $32: 0$ & $\mathrm{O}$ \\
\hline
\end{tabular}

\subsubsection{Gather}

Table 9.2 shows all Gather block ports. The Gather block retrieves all profiling data from the Tracers and sends it to the Control FPGA. Inputs Data_In and Event Done are the concatenated data of all Tracers Data_Out and Event_Done outputs, respectively. Output Fifo_Read_Tracers is the concatenated read signals for each Tracer FIFO. Output Data_Out connects to the Interchip and Valid_Data is enabled when that data is valid. Table 9.2 shows all Gather block ports. 
Table 9.3: Collector Interface

\begin{tabular}{|c|c|c|}
\hline Signal Name & MSB:LSB & I/O \\
\hline Clk & & I \\
\hline Interchip_Clk & & I \\
\hline Rst & $63: 0$ & I \\
\hline Cycle_Counter & N_FPGAS*33-1:0 & I \\
\hline Data_in & & I \\
\hline Valid_Data & $2: 0$ & I \\
\hline Number_of_Boards & $7: 0$ & I \\
\hline Myrank & $31: 0$ & O \\
\hline Mem_Cmd_Address & & O \\
\hline Mem_Cmd_RNW & & O \\
\hline Mem_Cmd_Valid & $31: 0$ & O \\
\hline Mem_Cmd_Tag & & I \\
\hline Mem_Cmd_Ack & $143: 0$ & O \\
\hline Mem_Wr_Din & $17: 0$ & O \\
\hline Mem_Wr_BE & $31: 0$ & I \\
\hline FSL_S_DATA (FROM_NETIF) & & I \\
\hline FSL_S_CTRL (FROM_NETIF) & & I \\
\hline FSL_S_EXISTS (FROM_NETIF) & & O \\
\hline FSL_S_READ (FROM_NETIF) & & O \\
\hline FSL_M_DATA_0 (FSL_SYNC) & $31: 0$ & O \\
\hline FSL_M_CTRL_0 (FSL_SYNC) & & O \\
\hline FSL_M_WRITE_0 (FSL_SYNC) & & I \\
\hline FSL_M_FULL_0 (FSL_SYNC) & & I \\
\hline FSL_M_DATA_1 (TO_NETIF) & $31: 0$ & I \\
\hline FSL_M_CTRL_1 (TO_NETIF) & & \\
\hline FSL_M_EXISTS_1 (TO_NETIF) & & \\
\hline FSL_M_READ_1 (TO_NETIF) & & \\
\hline & & \\
\hline
\end{tabular}

\subsubsection{Collector}

Table 9.3 shows all Collector block ports. The Collector block receives all profiling data from all the User FPGAs and from the Control FPGA Gather. Moreover, the Collector block also performs synchronization with all other boards. Due to this synchronization, the collector is connected to three FSLs: from the XAUI network interface (FROM_NETIF), from where the Collector removes all synchronization messages; to the network interface of board 1 (TO_NETIF), to where the Collector writes all messages read from the XAUI network interface that are not synchronization messages; and to the XAUI network Interface (FSL_SYNC), 
where the Collector sends all its synchronization messages. Both profiling packets and synchronization packets are saved in the DDR2 memory. 


\section{Bibliography}

[1] Xtreme Data Inc. http://www.xtremedatainc.com/.

[2] DRC Computer. http://www. drccomputer.com/.

[3] IBM. http://www-01.ibm.com/chips/techlib/techlib.nsf/ products/PowerPC_405_Embedded_Cores.

[4] Arun Patel, Christopher Madill, Manuel Saldaña, Christopher, Comis Régis Pomès, and Paul Chow. A scalable fpga-based multiprocessor. In In IEEE Symposium on FieldProgrammable Custom Computing Machines (FCCM'06), pages 111-120, April 2006.

[5] Manuel Saldaña and Paul Chow. Tmd-mpi: An MPI implementation for multiple processors across multiple fpgas. In In IEEE International Conference on Field-Programmable Logic and Applications (FPL 2006), pages 329-334, August 2006.

[6] Manuel Saldaña, Daniel Nunes, Emanuel Ramalho, and Paul Chow. Configuration and programming of heterogeneous multiprocessors on a multi-fpga system. In In 3rd International Conference on ReConFigurable Computing and FPGAs 2006 (ReConFig'06), September 2006.

[7] The MPI Forum. MPI: a message passing interface. In Supercomputing '93: Proceedings of the 1993 ACM/IEEE conference on Supercomputing, pages 878-883, New York, NY, USA, 1993. ACM Press. 
[8] Richard L. Graham and Timothy S.Woodall and Jeffrey M. Squyres. Open mpi: A flexible high performance mpi. In In Proceedings, 6th Annual International Conference on Parallel Processing and Applied Mathematics, Poznan, Poland, 2005.

[9] Chen Chang, John Wawrzynek, and Robert W. Brodersen. BEE2: A High-End Reconfigurable Computing System. IEEE Des. Test '05, 22(2):114-125, 2005.

[10] Seth Koehler, John Curreri, and Alan D. George. Performance analysis challenges and framework for high-performance reconfigurable computing. Parallel Computing, 34(45):217-230, 2008.

[11] Susan L., Graham Peter, B. Kessler, and Marshall McKusick. Gprof: A call graph execution profiler. In Proceeding of Department of the ACM SIGPLAN 1982 Symposium on Compiler Construction, Boston, MA, June 1982.

[12] Browne S., Deane C., Ho G., and Mucci P. Papi: A portable interface to hardware performance counters. In Proceedings of Department of Defense HPCMP Users Group Conference, June 1999.

[13] Xilinx. http://www.xilinx.com/.

[14] Altera. http://www.altera.com/.

[15] Lesley Shannon and Paul Chow. Maximizing system performance using reconfigurability to monitor system communications. In In International Conference on FieldProgrammable technology (FPT), pages 231-238, Brisbane, Australia, December 2004.

[16] W. Gropp and E. Lusk and N. Doss and A. Skjellum. A High-Performance, Portable Implementation of the MPI Message Passing Interface Standard. Parallel Computing, 22(6):789-828, 1996.

[17] Adam Leko and Hans Sherburne. MPE/jumpshot evaluation. 
[18] Hans Meuer, Erich Strohmaier, Jack Dongarra, and Horst Simon. "Top500 Supercomputer Sites". http: //www . top500 .org/.

[19] J. Dongarra, P. Luszczek, and A. Petitet. "The LINPACK Benchmark: Past, Present and Future". http://www. netlib.org/utk/people/JackDongarra/PAPERS/ hpl.pdf.

[20] J. J. Dongarra, C. B. Moler, J. R. Bunch, and G.W. Stewart. "LINPACK Users' Guide”. SIAM, 1979.

[21] C. L. Lawson and R. J. Hanson and D. R. Kincaid and F. T. Krogh. Basic Linear Algebra Subprograms for Fortran Usage. ACM Transactions on Mathematical Software (TOMS), 5(3):308-323, 1979.

[22] Emanuel Ramalho. The LINPACK Benchmark on a Multi-Core Multi-FPGA System, Department of Electrical \& Computer Engineering, University of Toronto, Master Thesis, 2008. 